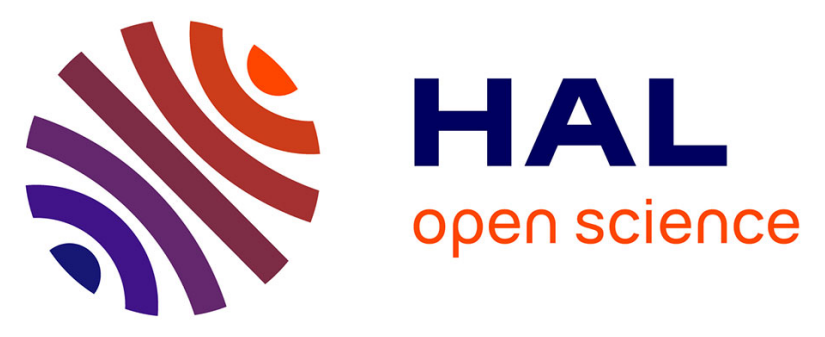

\title{
Regioselective Galactofuranosylation for the Synthesis of Disaccharide Patterns Found in Pathogenic Microorganisms
}

Laurent Legentil, Yari Cabezas, Olivier Tasseau, Charles Tellier, Franck Daligault, Vincent Ferrières

\section{To cite this version:}

Laurent Legentil, Yari Cabezas, Olivier Tasseau, Charles Tellier, Franck Daligault, et al.. Regioselective Galactofuranosylation for the Synthesis of Disaccharide Patterns Found in Pathogenic Microorganisms. Journal of Organic Chemistry, 2017, 82 (14), pp.7114-7122. 10.1021/acs.joc.7b00565 . hal-01578548

HAL Id: hal-01578548

https://hal-univ-rennes1.archives-ouvertes.fr/hal-01578548

Submitted on 13 Sep 2017

HAL is a multi-disciplinary open access archive for the deposit and dissemination of scientific research documents, whether they are published or not. The documents may come from teaching and research institutions in France or abroad, or from public or private research centers.
L'archive ouverte pluridisciplinaire $\mathbf{H A L}$, est destinée au dépôt et à la diffusion de documents scientifiques de niveau recherche, publiés ou non, émanant des établissements d'enseignement et de recherche français ou étrangers, des laboratoires publics ou privés. 


\title{
Regioselective galactofuranosylation for the synthesis of disaccharide patterns found in
} pathogenic microorganisms

\author{
Laurent Legentil, ${ }^{* a}$ Yari Cabezas, ${ }^{a}$ Olivier Tasseau, ${ }^{a}$ Charles Tellier, ${ }^{b}$ Franck Daligault ${ }^{b}$ and \\ Vincent Ferrières* ${ }^{\mathrm{a}}$
}

(a) Ecole Nationale Supérieure de Chimie de Rennes, CNRS, UMR 6226, 11 Allée de Beaulieu, CS 50837, 35708 Rennes Cedex 7, France

(b) Université de Nantes, UMR CNRS 6286, 2 Rue de la Houssinière, 44322 Nantes Cedex 3, France laurent.legentil@ensc-rennes.fr or vincent.ferrieres@ensc-rennes.fr

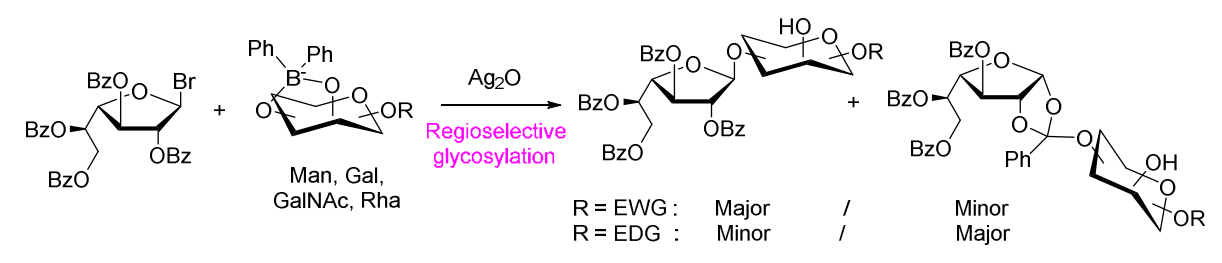

\begin{abstract}
Koenigs-Knorr glycosylation of acceptors with more than one free hydroxyl group by 2,3,5,6-tetrabenzoyl galactofuranosyl bromide was performed using diphenylborinic acid 2-aminoethyl ester (DPBA) as inducer of regioselectivity. High regioselectivity for the glycosylation on the equatorial hydroxyl group of the acceptor was obtained thanks to the transient formation of a borinate adduct of the corresponding 1,2-cis diol. Nevertheless formation of orthoester by-products hampered the efficiency of the method. Interestingly electron-withdrawing groups on $O-6$ or on $C-1$ of the acceptor displaced the reaction in favour of the desired galactofuranosyl containing disaccharide. The best yield was obtained for the furanosylation of $p$-nitrophenyl 6-O-acetyl mannopyranoside. Precursors of other disaccharides, found in the glycocalix of some pathogens, were synthesized according to the same protocol with yields ranging from 45 to $86 \%$. This is a good alternative for the synthesis of biologically relevant glycoconjugates.
\end{abstract}




\section{Introduction}

The significant discoveries on the organization of the cell, either from prokaryote or eukaryote origin, have pointed out the key role of the glycocalix, the complex layer of heteroand oligosaccharides that surrounds the cell wall. ${ }^{1-4}$ Even if most cells use common sugars to build this glycocalix, they manage to differentiate from each other thanks to the infinite possibilities of sequence and the nature of the linkages involved. Intriguingly some microorganisms incorporate carbohydrates in their furanose form rather than in their pyranose one in order to further differentiate themselves from other organisms. ${ }^{5,6}$ In particular Dgalactofuranose (D-Galf) containing glycoconjugates are found in large amount in pathogenic microorganisms like Mycobacteria or Leishmania but are absent in the mammalian kingdom. ${ }^{7}$ Owing to the key role played by such motifs in the virulence or survival of the parasite or bacteria, numerous groups have developed evolved synthetic pathways to access to such Galfcontaining oligosaccharides. ${ }^{8-13}$ Alternatively galactofuranosyl-transferases isolated from mycobacteria or furanosylhydrolases involved in the degradation of biomass have been used to obtain either oligomers of Gal $f^{14,15}$ or heterodisaccharides. ${ }^{16,17}$ These last strategies involve a minimum of protecting group manipulation as biocatalysts are able to selectively transfer a furanosyl entity on a specific position of an acceptor.

Attempt to mimic such selectivity remains the grail for glycochemists. Already, regioselective glycosylation of naked acceptors could be performed thanks to transient selective activation of one hydroxyl group against the others using for example dibutyltin(IV) oxide ${ }^{18,19}$ or aryl borinic $\operatorname{acid}^{20,21}$ as inducers of regioselectivity. More recently, Taylor and coworkers developed new diarylborinic acid derivatives for the regioselective acylation, ${ }^{22}$ alkylation ${ }^{23}$ or tosylation $^{24}$ of the secondary alcohol of various alkyl glycosides. They postulated that diarylborinic acid could form tetracoordinate adducts with 1,3-diols and 1,2-cis diols thus increasing the nucleophilicity of either the primary hydroxyl group or the least hindered 
equatorial one. The methodology was recently extended to the glycosylation of thiophenyl mannopyranoside using D-arabinofuranosyl methanesulfonate as a donor. ${ }^{25}$ It is the first example of the synthesis of furanosyl containing disaccharides obtained by this methodology and works remain to be done to apply such process to the synthesis of hexofuranosylcontaining conjugates.

After having proposed to work with unprotected thioimidates as donors, ${ }^{26}$ we now report on the use of diphenylborinic acid 2-aminoethyl ester (DPBA) to induce regioselectivity for galactofuranosylation of various glycosidic acceptors: D-mannopyranosides (D-Manp), Dgalactopyranoside $\quad(\mathrm{D}-\mathrm{Gal} p), \quad N$-acetyl-D-galactopyranoside $\quad$ (D-Gal $p$ NAc), $\quad$ Lrhamnopyranoside (L-Rhap) and D-Galf. The target compounds are disaccharidic parts of biomolecules anchored to the cell wall of pathogenic microorganisms (Figure 1), ${ }^{5,6}$ Representative examples include $\beta-(1 \rightarrow 3)$ and $(1 \rightarrow 6)$-D-Man $p$ linkage found in Aspergillus, Trypanosoma or Leishmania sp.; $\beta-(1 \rightarrow 3)-\mathrm{D}-\mathrm{Gal} p$ or $\beta-(1 \rightarrow 3)-\mathrm{D}-\mathrm{Gal} p$ NAc linkage identified in Bacteroides, Fibrobacter or Agelas sp. for the first one or Bacteroides and Shigella for the second one; $\beta-(1 \rightarrow 4)-$ L-Rha $p$ bond presented by Mycobacterium. ${ }^{6,27}$ Wide ligation diversity was also established between two D-Gal $f$ entities: $\beta-(1 \rightarrow 5)$ and $\beta-(1 \rightarrow 6)$ found in particular in Mycobacterium tuberculosis, Cryphonectria parasitica and Aspergillus. ${ }^{28}$

Some of these disaccharides linked the Gal $f$ non-reducing end to the most nucleophilic equatorial oxygen of the sugar at the reducing end. This oxygen is in addition in a 1,2-cis configuration with one of the proximal hydroxyl group. Such glycosidic bonds could therefore be selectively obtained thanks to glycosylation in presence of diarylborinic inducer. First the conditions of glycosylation were optimized with mannopyranosidic acceptor using peracylated galactofuranosyl bromide as donor. Different groups were introduced on the acceptor either on the primary hydroxyl group or at the anomeric position in order to investigate the influence of the inductive effect on the glycosylation. An attempt of 
rationalization of the results was performed thanks to $a b$ initio calculation. Finally the methodology was extended to the other relevant acceptors.

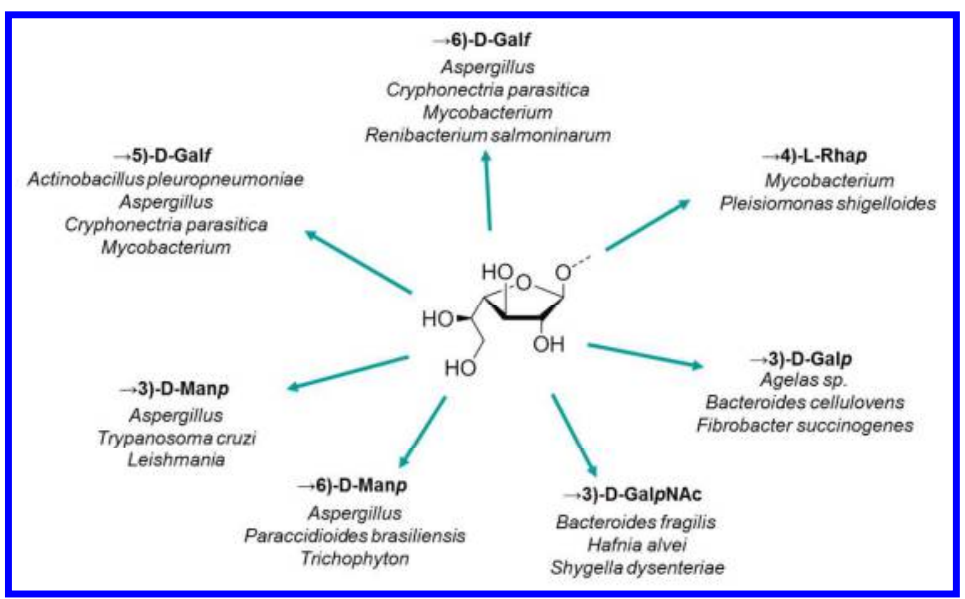

FIGURE 1. Natural occurrence of galactofuranose in some glycocalix of selected organisms.

\section{Results and discussion}

Our aim is to develop a regio- and stereoselective glycosylation to obtain Galf-containing disaccharides. The conditions developed by Taylor $^{29}$ were first applied on a model reaction between various Gal $f$ donors and Man $p$ acceptors (Figure 2). Different activating groups were introduced from peracylated derivatives of galactofuranose, namely the bromide, ${ }^{30}$ the thiopheny $^{31}$ or the trichloroacetimidate ${ }^{32}$ according to available protocols to give $\mathbf{1 a}, \mathbf{1 b}$ and 1c respectively. As for the acceptors, we decided to evaluate the influence of the protecting group on both positions $C-1$ and $O-6$. The primary position has to be masked anyway in order to avoid glycosylation at this position. Starting from commercially available $p$-nitrophenyl mannopyranoside ( $p$ NP Man $p$ ) 2a, the electron-donating group tert-butyldimethylsilyl was first introduced on position $O-6$ by action of the corresponding silylating agent $\mathrm{TBSCl}$ in presence of imidazole and DMAP with a moderate $40 \%$ yield. The electron-withdrawing group acetyl on the other hand was added using vinyl acetate in THF in presence of the supported lipase from Candida antartica (CAL-B). This last strategy was also applied to the 
octyl and thiotolyl $\alpha-\operatorname{Man} p$ 3a and $\mathbf{3 b}$ to obtain the corresponding 6-OAc mannoside $\mathbf{4 a}$ and 4b with $89 \%$ and $69 \%$ yields respectively.

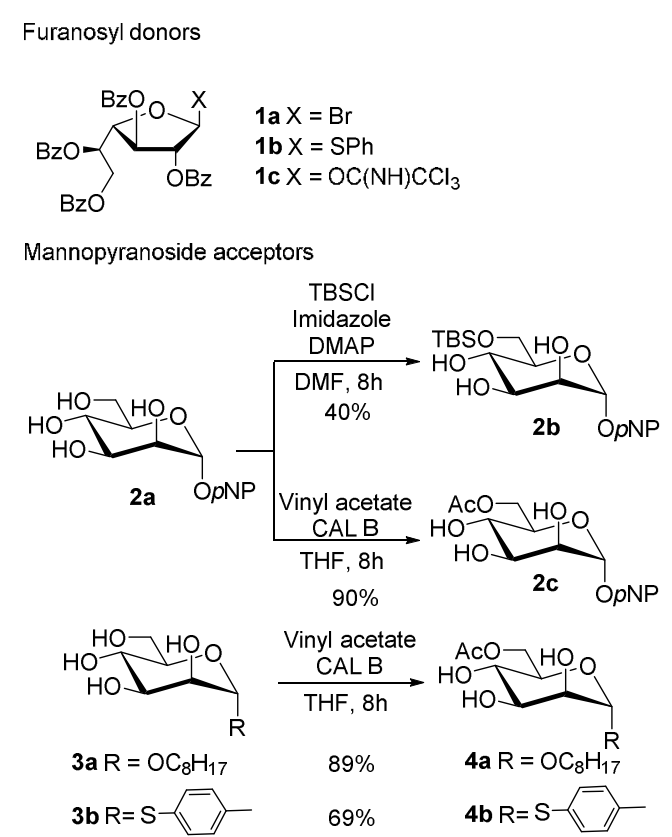

FIGURE 2. Building blocks used in the regioselective glycosylation.

With the donors $\mathbf{1}$ and acceptors $\mathbf{2}$ in hand, different attempts of glycosylation were implemented (Table 1). Following the findings of Taylor and coworkers, a catalytic amount of diphenylborinic acid 2-aminoethyl ester (DPBA) was used first (Entries 1, 3 to 7). The reactions proceeded in acetonitrile in presence of molecular sieves $4 \AA$ in order to limit the hydrolysis of the donors $\mathbf{1}$. These donors were used also in excess in the case where the hydrolysis is too high. With TBS Manp $\mathbf{2 b}$ as acceptor and bromide 1a as donor, the reaction proceeded quickly in presence of both silver oxide and the borinic acid to give the target disaccharide $\mathbf{5 b}$ in $36 \%$ yield (Entry 1). It was unambiguously characterized thanks to ${ }^{1} \mathrm{H}$ and ${ }^{13} \mathrm{C}$ NMR spectroscopy. The isolated disaccharide presented an anomeric proton at $5.55 \mathrm{ppm}$ associated with a carbon at $103.8 \mathrm{ppm}$. Coupling constant $J_{\mathrm{H} 1, \mathrm{H} 2}$ was almost zero, a typical value for an anomeric proton of a furanoside in 1,2-trans configuration. The regioisomery of the disaccharide was further confirmed by 2D-NMR HMBC experiments. A long range correlation between $\mathrm{C}_{\mathrm{Gal} f}-1$ and $\mathrm{H}_{\mathrm{Manp}}-3$ indicated a $(1 \rightarrow 3)$-glycosidic bond. The other product 
of the reaction, and the major one, was identified as the orthoester $6 \mathbf{b}$. It showed a characteristic signal in ${ }^{1} \mathrm{H}$ NMR at $6.47 \mathrm{ppm}$ associated with a carbon at $105.3 \mathrm{ppm}$. The related coupling constant $J_{\mathrm{H} 1, \mathrm{H} 2}$ reached $4.4 \mathrm{~Hz}$ thus indicating a 1,2-cis configuration. In addition ${ }^{13} \mathrm{C}$ NMR showed a carbon at 123.3 ppm assigned to the quaternary carbon of the orthoester function.

For comparative purpose, we tested the absence of DPBA (Entry 2) or of TBS on the primary hydroxyl group of the acceptor (Entry 3). Both conditions led to a complex mixture of products. As expected when no borinic acid was added the glycosylation occurred on different positions of the acceptor and the disaccharide $\mathbf{5 b}$ was isolated in only $10 \%$ yield. Also no protecting group on $O-6$ resulted in the concomitant glycosylation of both positions 3 and 6 , the two positions that were activated by the inducers of regioselectivity. ${ }^{29}$ In these conditions only trisaccharide 3,6-di- $O$-( $\beta$-D-Galf)-D-Man $p$ could be isolated.

Then we tried to isomerize the orthoester $\mathbf{6 b}$ into $\mathbf{5 a}$ by heating the reaction media or by the addition of a Lewis acid but only the degradation of the orthoester occurred. Alternatively another halogenophile, the silver triflate was used as promoter as it is known to avoid orthoester formation. However in these acidic conditions, the TBS group has been cleaved and again the trisaccharide 3,6-di- $O-(\beta-\mathrm{D}-\mathrm{Gal} f)$-D-Man $p$ was obtained. Therefore the silyl group was swapped by an acetyl one. After reaction between 1a and 2c (Entry 4), the major product remained the orthoester $\mathbf{6 c}$ and $\mathbf{5 c}$ was obtained only in $19 \%$ yield. As the acetyl group is tolerant to acidic condition, silver triflate was this time used successfully as promoter (Entry 5). It allowed to access to 5c with a better yield and no orthoester was isolated.

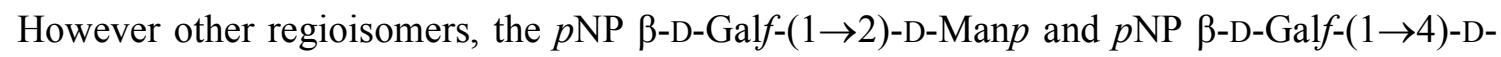
Man $p$ were formed as well. It confirms that acidic conditions are detrimental to the formation of the borinate complex. ${ }^{24}$ Finally, when other Gal $f$ donors $\mathbf{1 b}$ and $\mathbf{1 c}$ were used (Entries 6 and 7), the yield did not improve and the regioselectivity was poorer as previously reported. ${ }^{29}$ 
TABLE 1. Optimization of the conditions for the regioselective galactofuranosylation of pNP Man $p$ derivatives.

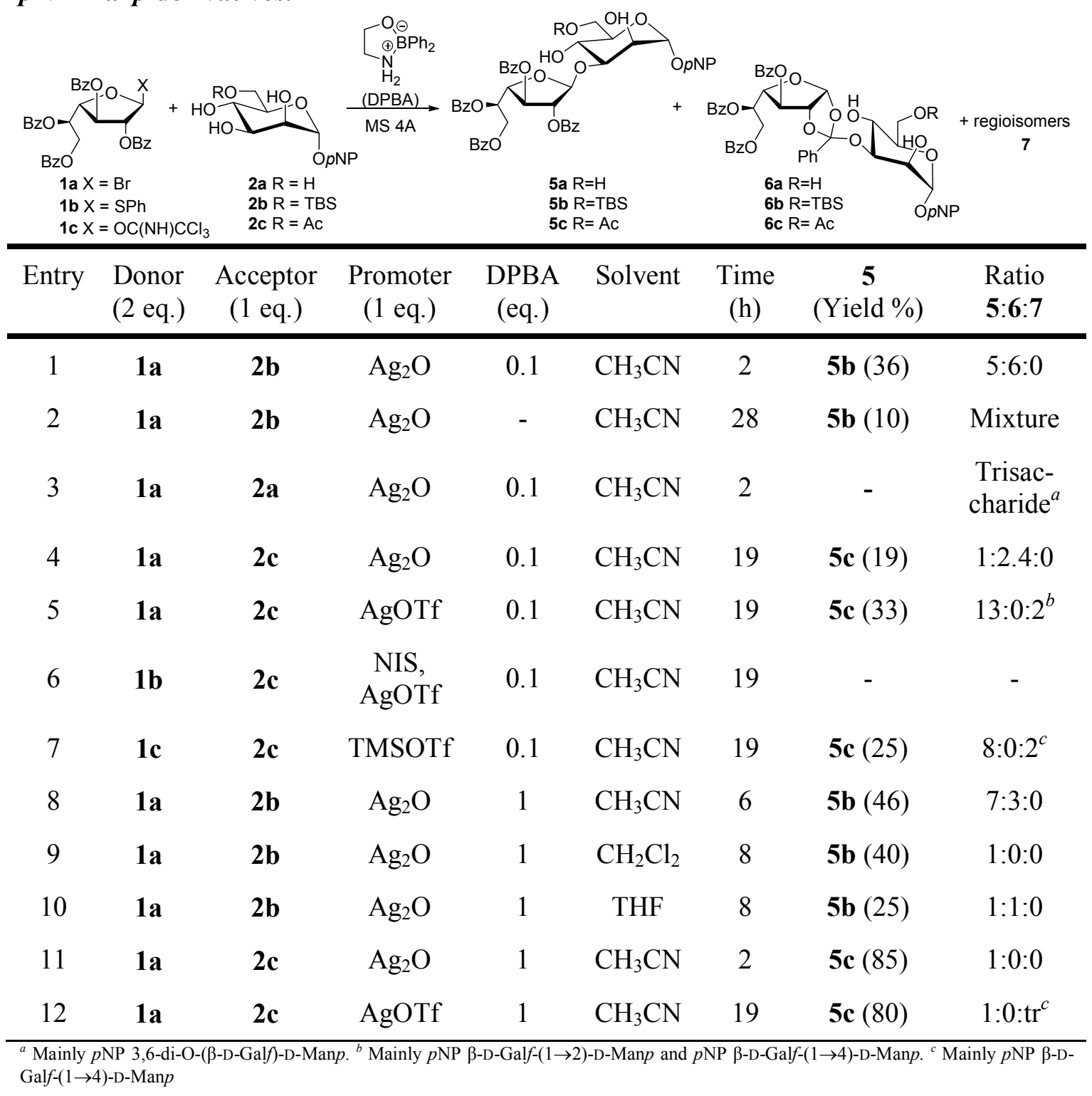

Interestingly, when a stoichiometric amount of DPBA was used, the yield greatly improved and no or trace amount of orthoester was formed (Entries 8 to 12). In addition the use of alternative solvents like THF or dichloromethane decreased either the yields or the selectivity (Entries 9 and 10). Also, the glycosylation of 6-O-TBS Manp 2b proceeded with lower yields than with 6-O-Ac Manp 2c, and a higher amount of orthoester $\mathbf{6 b}$ was isolated (Entries $8 v s$. 11). As for the promoter, the acidic AgOTf can be used but trace amount of regioisomers (mainly $\beta-(1 \rightarrow 4)-)$ contaminated again the disaccharide 5c (Entry 12$)$. The best result was 
obtained using the bromide 1a as donor, activated by one molar ratio of silver oxide, the 6-Oacetyl acceptor 2c, DPBA (one equivalent), in acetonitrile. Under these conditions, the $p$ NP $\beta$ D-Galf-(1 $\rightarrow 3)-\alpha-D-M a n p$ compound $\mathbf{5 c}$ was obtained in a quite worthy yield of $85 \%$ with no contamination by the orthoester (Entry 11).

Following this optimization, the methodology was then applied to 6-O-acetylated mannosidic acceptors $\mathbf{4 a}$ and $\mathbf{4 b}$ (Table 2) that differ on the nature of the substituent attached to the anomeric position. On one hand, the presence of the electron-donating group octyl at the anomeric position seemed to favour greatly the formation of the orthoester 9a (Entry 1). Solely the use of silver trifluoromethanesulfonate instead of silver oxide allowed isolating the target compound 8a with $57 \%$ yield (Entry 2). Once again, contamination by other regioisomers hampered the use of such acidic promoter. On the other hand, a completely different outcome arose with a thiotolyl group on $C$-1 of the mannose as the donor 1a easily glycosylated the position 3 of the mannoside $\mathbf{4 b}$. Formation of the orthoester $\mathbf{9 b}$ was low and no other regioisomers were isolated (Entry 3 and 4). It confirms the great influence of the electron-density of the substituent on both position $O-6$ and $C-1$.

TABLE 2. Extension to octyl and thiotolyl mannoside.

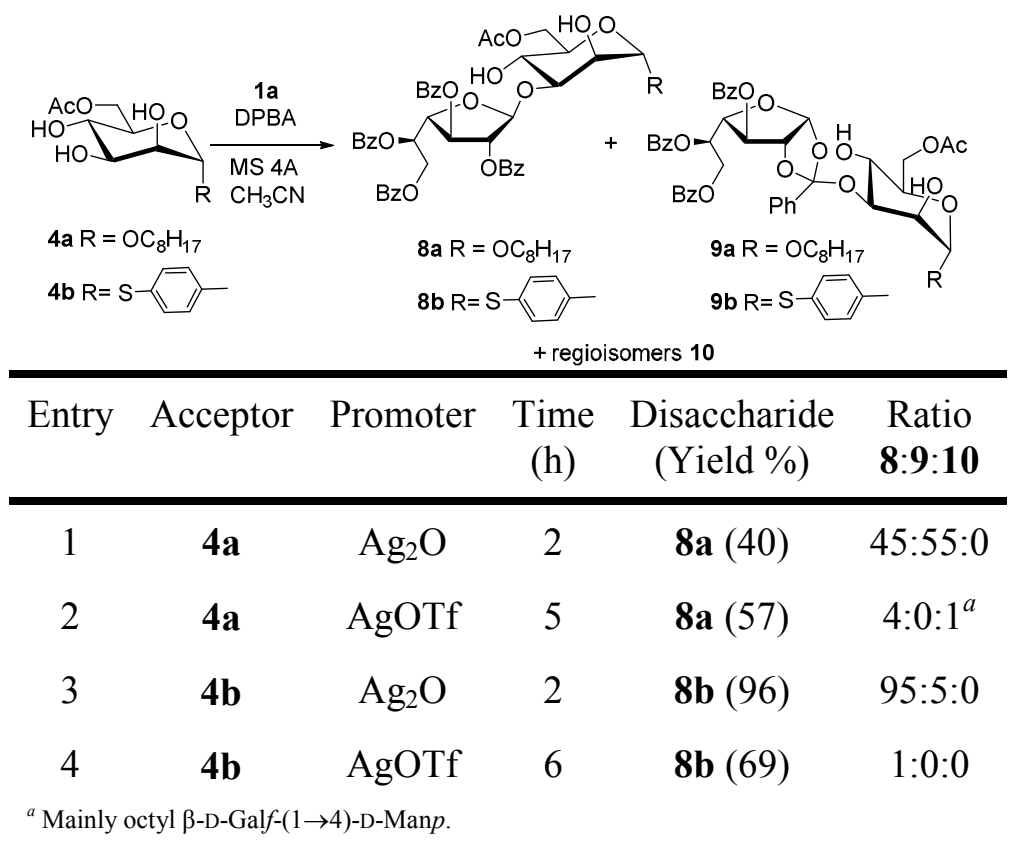


To explain these results, we decided to compare the difference of reactivity of the tested acceptors $\mathbf{2 b}, \mathbf{2 c}, \mathbf{4 a}$ and $\mathbf{4 b}$ in complex with diphenylborinic acid using DFT calculation in the gas-phase (B3LYP/6-31+G*) (Table 3 and Supporting Information).

\section{TABLE 3. Calculated Mulliken charges and Fukui indexes of diphenylborinate adduct}

of $2 b, 2 c, 4 a$ and $4 b\left(B 3 L Y P / 6-31+G^{*}\right)$.

\begin{tabular}{cccc}
\hline Entry & $\begin{array}{c}\text { Mannoside } \\
\text { acceptor }\end{array}$ & $\begin{array}{c}\text { Mulliken } \\
\text { charge at } O-3\end{array}$ & $f_{\mathrm{k}}$ at $O-3$ \\
\hline 1 & $\mathbf{2 b}$ & -0.35 & 0.180 \\
2 & $\mathbf{2 c}$ & -0.31 & 0.115 \\
3 & $\mathbf{4 a}$ & -0.32 & 0.135 \\
4 & $\mathbf{4 b}$ & -0.33 & 0.115
\end{tabular}

Surprisingly, the estimation of the Mulliken charges on the most reactive equatorial oxygen $O-3$ did not vary much between the different mannosides. Only the Fukui index $f_{\mathrm{k}}$, a measure of the strength of the nucleophile, ${ }^{33}$ decreased significantly with the presence of electronwithdrawing groups either on $O-6$ or on $C$-1. Interestingly, those molecules $\mathbf{2 c}$ and $\mathbf{4 b}$ also formed no or trace amount of orthoesters. On the contrary, electron-donating groups like the TBS (2b) or the alkyl (4a) one reinforced the nucleophilicity on $O-3$. Those acceptors generated after glycosylation the largest amount of orthoester. Orthoesters are known to form in various amounts during Koenigs-Knorr glycosylation. ${ }^{34}$ According to Taylor et al's work on pyranosyl bromide, ${ }^{29}$ the formation of such orthoester is not favoured because the reaction pathway follows a pure $\mathrm{S}_{\mathrm{N}} 2$ mechanism. Here, the presence of the bromide in a 1,2-trans configuration and the assistance of the benzoyl group on $O-2$ favour the transient acyloxonium specie and not the oxonium intermediate (Figure 3). The formation of such intermediate is in addition predominant in the furanose series. ${ }^{35}$ The attack of the nucleophile could then occur either at the quaternary carbon or at the anomeric one via an $\mathrm{S}_{\mathrm{N}} 2$ 
mechanism. Strong nucleophiles like $\mathbf{4 a}$ or $\mathbf{2 b}$ reacted first at the quaternary carbon and then at the anomeric position leading to a mixture of orthoester and disaccharide. The resulting orthoesters were stable enough to be isolated by silica gel chromatography. ${ }^{36}$ On the contrary, if less nucleophilic acceptors $\mathbf{2 c}$ and $\mathbf{4 b}$ were used, the attack occured only at the anomeric center to give the corresponding disaccharides $\mathbf{5 c}$ and $\mathbf{8 b}$. For the moment we are not able to decide if such selectivity is under kinetic or thermodynamic control. Nevertheless these results could be compared with the armed/disarmed effect linked with the nature of the substituent on the donor. Such activating/deactivating effects are however rarely reported when dealing with the acceptor and thus pave the way to a new understanding of the acceptor reactivity.

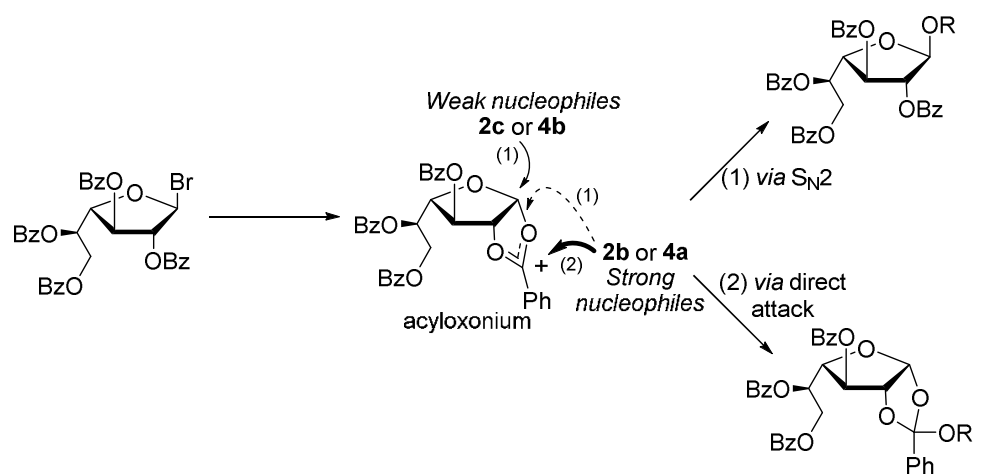

FIGURE 3. Mechanism of the formation of the orthoester and the disaccharide according to the nucleophilicity of the acceptor.

The same trend of reactivity was observed when the furanosylation assisted by DPBA was extended to the acceptors $p \mathrm{NP}$ galactopyranoside 12, $\mathrm{N}$-acetyl-galactosamine 14, Lrhamnoside 15 and galactofuranoside 16 (Table 4). The building blocks 12 and 14 were

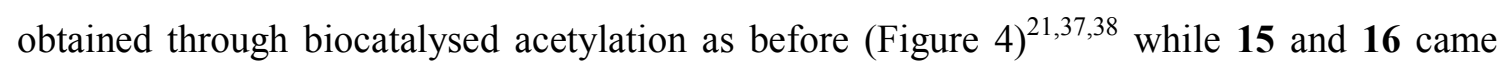
from commercial sources. 

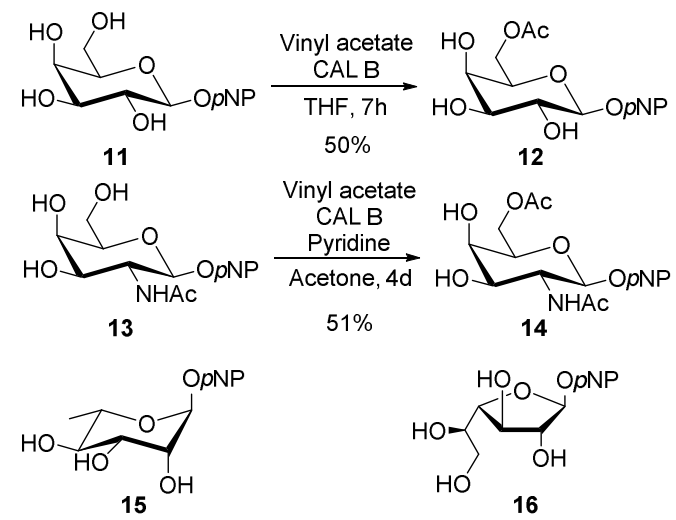

FIGURE 4. Acceptors used to obtain Galf-containing disaccharides found in various microorganisms.

On one hand 12 that bears electron-withdrawing groups on $O-6$ and $C-1$ and shows a low Fukui index at $O-3$, gave the corresponding disaccharide 17 with excellent yield and without formation of the orthoester (Entry 1). On the other hand the 6-deoxy-hexose $\mathbf{1 5}$ is rather nucleophilic and during the regioselective furanosylation catalysed by silver oxide, more than $30 \%$ of orthoester was formed (Entry 4). Alternative silver triflate protocol allowed to increase the yield for disaccharide 19 to $59 \%$ but the regioselectivity was decreased and $20 \%$ of the regiosiomer $p$ NP $\beta$-Galf-(1 $\rightarrow 4)$-L-Rha $p$ was obtained as well (Entry 5). Surprisingly, $p$ NP $N$-acetyl-galactosamine 14 whose calculated Fukui index was very low, gave the corresponding disaccharide 18 with poor yield (Entry 2). The amount of the corresponding orthoester was also low. The low nucleophilicity of $O-3$, due to the presence of the acetamide group on $C-2$ could explain the poor yield for the glycosylation. ${ }^{39}$ However $55 \%$ yield could be reached with silver triflate as promoter with the downside formation of regioisomers (Entry $3)$.

The last acceptor tested in the regioselective glycosylation was the $p$ NP Gal $f$ 16. In presence of the DPBA, we were expecting to form the diphenylborinate adduct between the $O-6$ and the $O-5$ and thus exacerbating the nucleophilicity of the primary hydroxyl group. Indeed the reaction between the bromide furanosyl 1a and $p$ NP Gal $f \mathbf{1 6}$ in presence of the borinic acid 
and silver oxide led quickly to the total conversion of the acceptor (Entry 6). The minor product of the reaction was then identified as the targeted $p \mathrm{NP} \beta-\mathrm{Gal} f-(1 \rightarrow 6)-\mathrm{Gal} f \mathbf{2 0}$ thanks to 2D-NMR experiments. The major one was the orthoester derivative isolated in $56 \%$ yield. This was expected as primary hydroxyl groups are much more reactive than secondary hydroxyl groups thus leading to high Fukui index and therefore to a high ratio of orthoester. It is worth to mention nevertheless that no other regioisomer was isolated which means that DPBA indeed formed a complex with $O-6$ and $O-5$ of the Gal $f$ moiety. Finally to prevent from the formation of the orthoester, silver triflate was used and allowed to obtain $\mathbf{2 0}$ with $45 \%$ yield in mixture with the regioisomer $p$ NP $\beta$-Galf-(1 $\rightarrow 5)$-Gal $f($ Entry 7$)$.

TABLE 4. Extension to relevant acceptors to obtain Galf-containing disaccharides.

\begin{tabular}{|c|c|c|c|c|c|}
\hline & $\begin{array}{l}17 \mathrm{R}=\mathrm{OH} \\
18 \mathrm{R}=\mathrm{NHAC}\end{array}$ & & 19 & 20 & \\
\hline Entry & Acceptor & $f_{\mathrm{k}}$ & Promoter & $\begin{array}{l}\text { Product } \\
\text { (Yield \%) }\end{array}$ & $\begin{array}{c}\text { By-product } \\
(\%)\end{array}$ \\
\hline 1 & 12 & $0.121^{a}$ & $\mathrm{Ag}_{2} \mathrm{O}$ & $17(86)$ & None \\
\hline 2 & \multirow[b]{2}{*}{14} & \multirow[b]{2}{*}{$0.101^{a}$} & $\mathrm{Ag}_{2} \mathrm{O}$ & $18(10)$ & Orthoester (37) \\
\hline 3 & & & AgOTf & $18\left(55^{c}\right)$ & 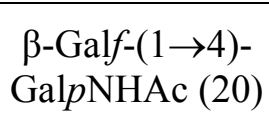 \\
\hline 4 & \multirow[b]{2}{*}{15} & \multirow[b]{2}{*}{$0.134^{a}$} & $\mathrm{Ag}_{2} \mathrm{O}$ & $19(45)$ & Orthoester (33) \\
\hline 5 & & & AgOTf & $19\left(59^{c}\right)$ & $\begin{array}{c}\beta \text {-Galf-(1 } \rightarrow 4)- \\
\text { L-Rhap }(20)\end{array}$ \\
\hline 6 & \multirow[b]{2}{*}{16} & \multirow[b]{2}{*}{$0.157^{b}$} & $\mathrm{Ag}_{2} \mathrm{O}$ & $20(12)$ & Orthoester (56) \\
\hline 7 & & & AgOTf & $20\left(45^{c}\right)$ & $\begin{array}{l}\beta \text {-Gal } f-(1 \rightarrow 5)- \\
\text { Gal } f(10)\end{array}$ \\
\hline
\end{tabular}

${ }^{a}$ Fukui index at $O-3 .{ }^{b}$ Fukui index at $O-6 .{ }^{c}$ As an inseparable mixture. Yield and ratio determined by ${ }^{1} \mathrm{H}$ NMR. 


\section{Conclusion}

As a conclusion, the high potential of diphenylborinic acid 2-aminoethyl ester as inducer of regioselective galactofuranosylation of various acceptors was confirmed. In this study we have validated the extension of Taylor's methodology from hexopyranosyl bromide to a 1,2trans hexofuranosyl bromide unable to react in a pure $\mathrm{S}_{\mathrm{N}} 2$ glycosylation pathway. When using silver oxide as promoter, only one regioisomer was formed as expected. High amount of orthoester however was formed when the $O-6$ or $C-1$ position of the acceptor possessed an electron-donating group. Silver triflate could be used instead but such an acidic catalyst destabilized the borinate adduct and the resulting regioselectivity dropped. The role of the substituents on the acceptor was partly explained thanks to ab initio calculation of the Fukui index of all borinate complexes. Electron-donating groups at primary and anomeric positions significantly increased the Fukui indexes and borinate adducts with high Fukui index on the equatorial oxygen generated the greater amount of orthoester. This is a confirmation that the electron-density of the substituent at the anomeric position of the acceptor can modulate the glycosylation reaction. Finally, despite these limitations, we managed to obtain quickly and efficiently galactofuranosyl containing disaccharides found in the glycocalix of pathogenic microorganisms. The minimum protecting group manipulation and the simplicity of the method represent an attractive alternative to biocatalysed process that still suffer from too strong specificity and low conversion yields.

\section{Experimental Section}

\section{General Experimental Details}

All reactions were carried out in oven-dried glassware. All reagents were purchased from commercial sources and were used without further purification unless noted. Dried acetonitrile, dichloromethane and THF were purchased sealed on molecular sieves. Unless 
otherwise stated, all reactions were monitored by TLC on Silica Gel $60 \mathrm{~F}_{254}$. TLC spots were detected under $254 \mathrm{~nm}$ UV-light or by staining with cerium ammonium molybdate solution. Column chromatography was performed on Silica Gel $(25$ or $50 \mu \mathrm{m})$. Optical rotations were measured at $20{ }^{\circ} \mathrm{C}$ on a Perkin-Elmer 341 polarimeter. NMR spectra were recorded at 400 $\mathrm{MHz}$ for ${ }^{1} \mathrm{H}$ and $100 \mathrm{MHz}$ for ${ }^{13} \mathrm{C}$. Chemical shifts are given in $\delta$ units (ppm) and referenced to either $\mathrm{CDCl}_{3}$ or $\mathrm{CD}_{3} \mathrm{OD}$. Coupling constants $J$ were calculated in Hertz (Hz). Proton and carbon NMR peaks were unambiguously assigned by COSY (double quantum filtered with gradient pulse for selection), HSQC (gradient echo-anti echo selection and shape pulse) and HMBC (echo-anti echo gradient selection, magnitude mode) correlation experiments. For each isolated oligosaccharide, the reducing end (bearing $p \mathrm{NP}$ or alkyl chain) was quoted as "a", and the letter increased toward the non-reducing end (for example the sugar after was quoted as "b"). High Resolution Masses were ) were recorded in positive mode using direct Electrospray ionization on a Waters Q-Tof 2 spectrometer.

4-Nitrophenyl 6-O-tert-butyldimethylsilyl-a-D-mannopyranoside (2b): To a solution of 4Nitrophenyl $\alpha$-D-mannopyranoside 2a (1.5 g, $4.98 \mathrm{mmol})$ in DMF (30 $\mathrm{mL})$ were added imidazole (1.02 g, $14.94 \mathrm{mmol})$, a catalytic amount of DMAP (30 mg, $0.25 \mathrm{mmol})$ followed by TBSCl (901 mg, $5.98 \mathrm{mmol})$. The reaction mixture was stirred at room temperature until no evolution was observed through TLC monitoring $(8 \mathrm{~h})$. The reaction mixture was diluted into $\mathrm{CH}_{2} \mathrm{Cl}_{2}(100 \mathrm{~mL})$ and washed with water $(100 \mathrm{~mL})$. The organic layer was washed twice with a saturated aq $\mathrm{NH}_{4} \mathrm{Cl}$ solution, water and brine. The resulting organic layer was dried with $\mathrm{MgSO}_{4}$, filtered and concentrated under reduced pressure. The remaining silane was removed by submitting the crude material to vacuum during several hours. The 6-O-protected mannopyranoside $\mathbf{2 b}$ was obtained as a white solid ( $829 \mathrm{mg}, 40 \%$ ) and could be used without further purification. $[\alpha]_{\mathrm{D}}{ }^{20}+115(\mathrm{c} 1, \mathrm{MeOH}) .{ }^{1} \mathrm{H} \mathrm{NMR}\left(\mathrm{CD}_{3} \mathrm{OD}\right): \delta_{\mathrm{H}} 8.20(2 \mathrm{H}, \mathrm{d}, J=9.3 \mathrm{~Hz}$, $\left.\mathrm{H}_{\mathrm{Ar}}\right), 7.28\left(2 \mathrm{H}, \mathrm{d}, J=9.3 \mathrm{~Hz}, \mathrm{H}_{\mathrm{Ar}}\right), 5.64\left(1 \mathrm{H}, \mathrm{d}, J_{1,2}=1.8 \mathrm{~Hz}, \mathrm{H}-1\right), 4.04\left(1 \mathrm{H}, \mathrm{dd}, J_{2,3}=3.4\right.$ 
Hz, H-2), $3.92\left(1 \mathrm{H}, \mathrm{dd}, J_{6,5}=2 \mathrm{~Hz}, J_{6,6^{\prime}}=11.2 \mathrm{~Hz}, \mathrm{H}-6\right), 3.87\left(1 \mathrm{H}, \mathrm{dd}, J_{3,4}=9.3 \mathrm{~Hz}, \mathrm{H}-3\right)$, $3.74\left(1 \mathrm{H}, \mathrm{dd}, J_{6}, 5=6.8 \mathrm{~Hz}, \mathrm{H}-6\right.$ '), $3.65\left(1 \mathrm{H}\right.$, app. t, $\left.J_{4,5}=9.5 \mathrm{~Hz}, \mathrm{H}-4\right), 3.50(1 \mathrm{H}, \mathrm{ddd}, \mathrm{H}-5)$, $0.8\left(9 \mathrm{H}, \mathrm{s}, \mathrm{C}\left(\mathrm{CH}_{3}\right)_{3}\right), 0.02\left(3 \mathrm{H}, \mathrm{s}, \mathrm{OSi}\left(\mathrm{CH}_{3}\right)_{2}\right), 0.00\left(3 \mathrm{H}, \mathrm{s}, \mathrm{OSi}\left(\mathrm{CH}_{3}\right)_{2}\right) .{ }^{13} \mathrm{C} \mathrm{NMR}\left(\mathrm{CD}_{3} \mathrm{OD}\right):$ $\delta_{\mathrm{C}} 162.7\left(\mathrm{C}_{\mathrm{Ar}}\right), 143.8\left(\mathrm{C}_{\mathrm{Ar}}\right), 126.7\left(\mathrm{C}_{\mathrm{Ar}}\right), 118.0\left(\mathrm{C}_{\mathrm{Ar}}\right), 100.0(\mathrm{C}-1), 76.6(\mathrm{C}-5), 72.4(\mathrm{C}-3), 71.5$ (C-2), $68.4(\mathrm{C}-4), 64.3(\mathrm{C}-6), 26.3\left[\mathrm{SiC}\left(\mathrm{CH}_{3}\right)_{3}\right], 19.1\left[\mathrm{SiC}\left(\mathrm{CH}_{3}\right)_{3}\right],-5.15\left[\mathrm{OSi}\left(\mathrm{CH}_{3}\right)_{2}\right],-5.17$ [OSi $\left.\left(\mathrm{CH}_{3}\right)_{2}\right]$. HRMS (ESI/Q-TOF) m/z: [M+Na $]^{+} \mathrm{Calcd}$ for $\mathrm{C}_{18} \mathrm{H}_{29} \mathrm{NO}_{8} \mathrm{SiNa}$ 438.1560; Found 438.1560 .

General procedure for the regioselective acetylation of pyranosides: A solution $(0.1 \mathrm{M})$ of 4-nitrophenyl glycopyranoside in THF was heated to $45{ }^{\circ} \mathrm{C}$ until complete dissolution. Then vinyl acetate ( 3 equiv.) and Novozym ${ }^{\circledR} 435$ were added (Lipase acrylic resin Candida antartica from Novozymes) in a w/w ratio 1:1.2 [glycoside : immobilized enzyme]. The reaction mixture was stirred at $45^{\circ} \mathrm{C}$ until complete conversion of starting material $(\approx 7 \mathrm{~h})$. The enzyme was filtered off and rinsed with $\mathrm{MeOH}$. The resulting filtrate was then concentrated under reduced pressure and purified by column chromatography on silica gel (DCM/MeOH ratio 95:5).

4-Nitrophenyl 6-O-acetyl- $\alpha$-D-mannopyranoside (2c): Synthesized according to general procedure from $p \mathrm{NP} \alpha$-D-mannopyranoside $\mathbf{2 a}(1.0 \mathrm{~g}, 3.32 \mathrm{mmol})$, vinyl acetate $(918 \mu \mathrm{L}, 9.96$ mmol) and Novozym ${ }^{\circledR} 435(1.2 \mathrm{~g})$ to afford $2 \mathrm{c}$ as a pale yellow solid $(1.03 \mathrm{~g}, 90 \%) .[\alpha]_{\mathrm{D}}^{20}+$ 128 (c 1, MeOH). ${ }^{1} \mathrm{H}$ NMR $\left(\mathrm{CD}_{3} \mathrm{OD}\right): \delta_{\mathrm{H}} 8.24\left(2 \mathrm{H}, \mathrm{d},{ }^{3} J=9.3 \mathrm{~Hz}, \mathrm{H}_{\mathrm{Ar}}\right), 7.27\left(2 \mathrm{H}, \mathrm{d}, \mathrm{H}_{\mathrm{Ar}}\right)$, $5.63\left(1 \mathrm{H}, \mathrm{d}, J_{1,2}=1.7 \mathrm{~Hz}, \mathrm{H}-1\right), 4.33\left(1 \mathrm{H}, \mathrm{dd}, J_{6,6^{\prime}}=11.8 \mathrm{~Hz}, J_{6 \mathrm{a}, 5}=2 \mathrm{~Hz}, \mathrm{H}-6\right), 4.19(1 \mathrm{H}, \mathrm{dd}$, $J_{6^{\prime}, 5}=6.4, \mathrm{H}-6$ '), $4.05\left(1 \mathrm{H}, \mathrm{dd}, J_{2,3}=3.4 \mathrm{~Hz}, \mathrm{H}-2\right), 3,88\left(1 \mathrm{H}, \mathrm{dd}, J_{3,4}=9 \mathrm{~Hz}, \mathrm{H}-3\right), 3.73(1 \mathrm{H}$, app. t, $\left.J_{4,5}=9.8 \mathrm{~Hz}, \mathrm{H}-4\right), 3.61(1 \mathrm{H}, \mathrm{ddd}, \mathrm{H}-5), 1.94\left(3 \mathrm{H}, \mathrm{s}, \mathrm{CH}_{3} \mathrm{CO}\right) .{ }^{13} \mathrm{C} \mathrm{NMR}\left(\mathrm{CD}_{3} \mathrm{OD}\right): \delta_{\mathrm{C}}$ $172.7(\mathrm{CO}), 162.6\left(\mathrm{C}_{\mathrm{Ar}}\right), 144.0\left(\mathrm{C}_{\mathrm{Ar}}\right), 126.7\left(\mathrm{C}_{\mathrm{Ar}}\right), 117.9\left(\mathrm{C}_{\mathrm{Ar}}\right), 100(\mathrm{C}-1) ; 73.5(\mathrm{C}-4), 72.2(\mathrm{C}-$ 3), 71.5 (C-2), 68.3 (C-5), 64.8 (C-6), $20.7\left(\mathrm{CH}_{3} \mathrm{CO}\right)$. HRMS (ESI/Q-TOF) m/z: $[\mathrm{M}+\mathrm{Na}]^{+}$ Calcd for $\mathrm{C}_{14} \mathrm{H}_{17} \mathrm{NO}_{9} \mathrm{Na} 366.0801$; Found 366.0797. 
Octyl 6-O-acetyl-a-D-mannopyranoside (4a): Synthesized according to general procedure from octyl $\alpha$-D-mannopyranoside $\mathbf{3 a}^{40}$ (300 mg, $\left.1 \mathrm{mmol}\right)$, vinyl acetate $(284 \mu \mathrm{L}, 3.31 \mathrm{mmol})$ and Novozym ${ }^{\circledR} 435(400 \mathrm{mg})$ to afford $2 \mathrm{c}$ as a colorless oil $(305 \mathrm{mg}, 90 \%) \cdot[\alpha]_{\mathrm{D}}^{20}+43$ (c 1 , $\mathrm{MeOH}) .{ }^{1} \mathrm{H}$ NMR $\left(\mathrm{CD}_{3} \mathrm{OD}\right): \delta_{\mathrm{H}} 4.70\left(1 \mathrm{H}, \mathrm{d}, J_{1,2}=1.6 \mathrm{~Hz}, \mathrm{H}-1\right), 4.39\left(1 \mathrm{H}, \mathrm{dd}, J_{6,6},=11.7 \mathrm{~Hz}\right.$, $\left.J_{6,5}=2.0 \mathrm{~Hz}, \mathrm{H}-6\right), 4.20\left(1 \mathrm{H}, \mathrm{dd}, J_{6^{\prime}, 5}=6.4 \mathrm{~Hz}, \mathrm{H}-6^{\circ}\right), 3.79\left(1 \mathrm{H}, \mathrm{dd}, J_{2,3}=3.2 \mathrm{~Hz}, \mathrm{H}-2\right), 3.73-$ $3.65\left(3 \mathrm{H}, \mathrm{m}, \mathrm{H}-5, \mathrm{H}-3, \mathrm{OCH}_{2}\right), 3.60\left(1 \mathrm{H}, \mathrm{t}, J_{4,5}=9.4 \mathrm{~Hz}, \mathrm{H}-4\right), 3.42\left(1 \mathrm{H}, \mathrm{dt},{ }^{2} J=9.6 \mathrm{~Hz},{ }^{3} J=\right.$ $\left.6.3 \mathrm{~Hz}, \mathrm{OCH}_{2}\right), 2.06\left(3 \mathrm{H}, \mathrm{s}, \mathrm{COCH}_{3}\right), 1.66-1.54\left(2 \mathrm{H}, \mathrm{m}, \mathrm{OCH}_{2} \mathrm{CH}_{2}\right), 1.45-1.27(10 \mathrm{H}, \mathrm{m}$, $\left.\left(\mathrm{CH}_{2}\right)_{5} \mathrm{CH}_{3}\right), 0.91\left(3 \mathrm{H}, \mathrm{t},{ }^{3} \mathrm{~J}=6.8 \mathrm{~Hz}, \mathrm{CH}_{2} \mathrm{CH}_{3}\right) .{ }^{13} \mathrm{C}$ NMR $\left(\mathrm{CD}_{3} \mathrm{OD}\right): \delta_{\mathrm{C}} 172.8(\mathrm{CO}), 101.7$ (C-1), 72.6 (C-3), 72.1, 72.1 (C-2, C-5), 68.7, 68.7 (C-4, $\left.\mathrm{OCH}_{2}\right), 65.3$ (C-6), 33.0, 30.6, 30.5, 30.4, 27.4, 23.7[( $\left.\left(\mathrm{CH}_{2}\right)_{5} \mathrm{CH}_{3}\right], 20.8\left(\mathrm{COCH}_{3}\right), 14.4\left(\mathrm{CH}_{2} \mathrm{CH}_{3}\right)$. HRMS (ESI/Q-TOF) m/z: $[\mathrm{M}+\mathrm{Na}]^{+}$Calcd for $\mathrm{C}_{16} \mathrm{H}_{30} \mathrm{O}_{7} \mathrm{Na} 357.18837$; Found 357.1882.

Thiotolyl 6-O-acetyl- $\alpha$-D-mannopyranoside (4b): Synthesized according to general procedure from thiotolyl $\alpha$-D-mannopyranoside $\mathbf{3 b}^{41}$ (780 mg, $2.7 \mathrm{mmol}$ ), vinyl acetate (750 $\mu \mathrm{L}, 8.2 \mathrm{mmol})$ and Novozym ${ }^{\circledR} 435(1 \mathrm{~g})$ to afford $4 \mathbf{b}$ as a colorless oil $(624 \mathrm{mg}, 69 \%)$. $[\alpha]_{\mathrm{D}}^{20}$ $+244(\mathrm{c} 1.25, \mathrm{MeOH}) .{ }^{1} \mathrm{H}$ NMR $\left(\mathrm{CD}_{3} \mathrm{OD}\right): \delta_{\mathrm{H}} 7.40\left(2 \mathrm{H}, \mathrm{d},{ }^{3} J=8.1 \mathrm{~Hz}, \mathrm{H}_{\mathrm{Ar}}\right), 7.14(2 \mathrm{H}, \mathrm{d}$, $\left.\mathrm{H}_{\mathrm{Ar}}\right), 5.35\left(1 \mathrm{H}, \mathrm{d}, J_{1,2}=1.5 \mathrm{~Hz}, \mathrm{H}-1\right), 4.43-4.35$ (1H, m, H-6), 4.29-4.20 (2H, m, H-5, H-6'), $4.07\left(1 \mathrm{H}, \mathrm{dd}, J_{2,3}=2.9 \mathrm{~Hz}, \mathrm{H}-2\right), 3.72-3.64(2 \mathrm{H}, \mathrm{m}, \mathrm{H}-3, \mathrm{H}-4), 2.32\left(3 \mathrm{H}, \mathrm{s}, \mathrm{CH}_{3}\right), 2.00(3 \mathrm{H}, \mathrm{s}$, $\left.\mathrm{COCH}_{3}\right) .{ }^{13} \mathrm{C}$ NMR $\left(\mathrm{CD}_{3} \mathrm{OD}\right): \delta_{\mathrm{C}} 172.7(\mathrm{CO}), 139.0,133.5,131.7,130.7\left(\mathrm{C}_{\mathrm{Ar}}\right), 90.3(\mathrm{C}-1)$, 73.4 (C-2), 73.0, 72.9 (C-4, C-5), 69.0 (C-3), $65.0(\mathrm{C}-6), 21.1\left(\mathrm{CH}_{3}\right), 20.8\left(\mathrm{COCH}_{3}\right)$. HRMS (ESI/Q-TOF) m/z: [M+Na] ${ }^{+}$Calcd for $\mathrm{C}_{15} \mathrm{H}_{20} \mathrm{O}_{6} \mathrm{SNa} 351.08728$; Found 351.0872.

4-Nitrophenyl 6-O-acetyl- $\beta$-D-galactopyranoside (12): Synthesized according to general procedure from $p$ NP $\beta$-D-galactopyranoside $11(100 \mathrm{mg}, 0.3 \mathrm{mmol})$, vinyl acetate $(100 \mu \mathrm{L}$, $1 \mathrm{mmol})$ and Novozym ${ }^{\circledR} 435(120 \mathrm{mg})$ to afford 12 as a colorless oil (56 mg, 50\%). ${ }^{1} \mathrm{H}$ NMR $\left(\mathrm{CD}_{3} \mathrm{OD}+\mathrm{CDCl}_{3}\right): \delta_{\mathrm{H}} 8.20\left(2 \mathrm{H}, \mathrm{d},{ }^{3} J=9.3 \mathrm{~Hz}, \mathrm{H}_{\mathrm{Ar}}\right), 7.19\left(2 \mathrm{H}, \mathrm{d}, \mathrm{H}_{\mathrm{Ar}}\right), 4.99\left(1 \mathrm{H}, \mathrm{d}, J_{1,2}=7.7\right.$ $\mathrm{Hz}, \mathrm{H}-1), 4.34\left(1 \mathrm{H}, \mathrm{dd}, J_{6,6^{\prime}}=11.5 \mathrm{~Hz}, J_{6,5}=7.8 \mathrm{~Hz}, \mathrm{H}-6\right), 4.25\left(1 \mathrm{H}, \mathrm{dd}, J_{6^{\prime}, 5}=4.5 \mathrm{~Hz}, \mathrm{H}-6^{\circ}\right)$, 
3.99-3.91 (1H, m, H-5), $3.90\left(1 \mathrm{H}, \mathrm{dt}, J_{4,3}=3.5 \mathrm{~Hz}, J_{4,5}=0.9 \mathrm{~Hz}, \mathrm{H}-4\right), 3.85\left(1 \mathrm{H}, \mathrm{dd}, J_{2,3}=9.7\right.$

$\mathrm{Hz}, \mathrm{H}-2), 3.61(1 \mathrm{H}, \mathrm{dd}, \mathrm{H}-3), 2.07\left(3 \mathrm{H}, \mathrm{s}, \mathrm{CH}_{3}\right) .{ }^{13} \mathrm{C}$ NMR $\left(\mathrm{CD}_{3} \mathrm{OD}\right): \delta_{\mathrm{C}} 172.2(\mathrm{CO}), 163.4$, 143.5, 126.3, $117.4\left(\mathrm{C}_{\mathrm{Ar}}\right), 101.6(\mathrm{C}-1), 74.1$ (C-3, C-5), $71.4(\mathrm{C}-2), 69.5(\mathrm{C}-4), 64.4(\mathrm{C}-6)$, $20.9\left(\mathrm{CH}_{3}\right)$. HRMS (ESI/Q-TOF) m/z: $[\mathrm{M}+\mathrm{Na}]^{+}$Calcd for $\mathrm{C}_{14} \mathrm{H}_{17} \mathrm{NO}_{9} \mathrm{Na} 366.0801$; Found 366.0804 .

4-Nitrophenyl 6-O-acetyl-2-deoxy- $\boldsymbol{\beta}$-D- $\mathrm{N}$-acetylgalactosamine (14): Synthesized according to described procedure ${ }^{38}$ from $p$ NP $\beta$-D- $N$-acetylgalactosamine 13 (280 mg, $0.8 \mathrm{mmol}$ ), vinyl acetate $(10 \mathrm{~mL})$ Novozym ${ }^{\circledR} 435(350 \mathrm{mg})$ in a mixture of acetone/pyridine $(29 / 21 \mathrm{~mL})$ to afford 14 as a white solid (161 mg, 51\%). Spectroscopic data were in accordance with previous report.

General procedure for the 2-ADPB-assisted glycosidic coupling: Bromide donor $\mathbf{1 a}^{30}(2$ equiv.), acceptor (1 equiv.) and 2-aminoethyl diphenylborinate (2-DPBA, 1 equiv.) were suspended in dry $\mathrm{CH}_{3} \mathrm{CN}(40 \mathrm{mM})$ with activated $4 \AA$ molecular sieves $(100 \mathrm{mg} / \mathrm{mL})$ and under a nitrogen atmosphere. The mixture was stirred for 30 min and $\mathrm{Ag}_{2} \mathrm{O}$ (1 equiv.) was added. Alternatively, the mixture was protected from light and AgOTf (1 equiv.) was used. After stirring at room temperature, when no evolution was observed through TLC monitoring, the reaction mixture was diluted with EtOAc and filtered through a plug of Celite. In the case of AgOTf-promoted reactions, a few drops of $\mathrm{Et}_{3} \mathrm{~N}$ were added to neutralize the reaction prior to dilution and filtration. The filtrate was concentrated under reduced pressure and the resulting crude material was purified by column chromatography on silica gel (cyclohexane/ ethyl acetate ratio 4:1).

\section{4-Nitrophenyl}

2,3,5,6-tetra- $O$-benzoyl- $\beta$-D-galactofuranosyl-( $1 \rightarrow 3)$-6-O-tertbutyldimethylsilyl-a-D-mannopyranoside (5b): Synthesized according to general procedure starting from 6-O-TBS-mannopyranoside $2 \mathbf{b}(63 \mathrm{mg}, 0.15 \mathrm{mmol})$, galactofuranosyl bromide 
$1 \mathrm{a}(200 \mathrm{mg}, 0.30 \mathrm{mmol}), 2-\mathrm{DPBA}(34 \mathrm{mg}, 0.15 \mathrm{mmol})$ and $\mathrm{Ag}_{2} \mathrm{O}$ (35 mg, $0.15 \mathrm{mmol}$ ). Reaction reached completion after $6 \mathrm{~h}$. Purification by column chromatography on silica gel afforded 5b (70 $\mathrm{mg}, 46 \%)$ as a white solid. Further elution allowed isolating the corresponding orthoester $\mathbf{6 b}$ (37 mg, 24\%).

5b: $[\alpha]_{\mathrm{D}}{ }^{20}+12\left(\mathrm{c} 1, \mathrm{CH}_{2} \mathrm{Cl}_{2}\right) .{ }^{1} \mathrm{H} \mathrm{NMR}\left(\mathrm{CDCl}_{3}\right): \delta_{\mathrm{H}} 8.19\left(2 \mathrm{H}, \mathrm{d}, J=9.3 \mathrm{~Hz}, \mathrm{H}_{\mathrm{Ar}}\right), 8.10,8.04$, 7.99, $7.93\left(8 \mathrm{H}, 4 \mathrm{dd}, J=8.3 \mathrm{~Hz}, J=1.2 \mathrm{~Hz}, \mathrm{H}_{\mathrm{Ar}}\right), 7.61-7.29\left(12 \mathrm{H}, \mathrm{m}, \mathrm{H}_{\mathrm{Ar}}\right), 7.15\left(2 \mathrm{H}, \mathrm{d}, \mathrm{H}_{\mathrm{Ar}}\right)$, 6.03-5.98 (1H, m, H-5b), $5.76\left(1 \mathrm{H}, \mathrm{dd}, J_{3 \mathrm{~b}, 4 \mathrm{~b}}=5.8 \mathrm{~Hz}, \mathrm{~J}_{3 \mathrm{~b}, 2 \mathrm{~b}}=2 \mathrm{~Hz}, \mathrm{H}-3 \mathrm{~b}\right), 5.70\left(1 \mathrm{H}, \mathrm{d}, J_{1 \mathrm{a}, 2 \mathrm{a}}\right.$ $=1.5 \mathrm{~Hz}, \mathrm{H}-1 \mathrm{a}), 5.55(1 \mathrm{H}, \mathrm{bs}, \mathrm{H}-1 \mathrm{~b}), 5.49\left(1 \mathrm{H}, \mathrm{dd}, J_{2 \mathrm{~b}, 1 \mathrm{~b}}=0.7 \mathrm{~Hz}, \mathrm{H}-2 \mathrm{~b}\right), 4.95\left(1 \mathrm{H}, \mathrm{dd}, J_{4 \mathrm{~b}, 5 \mathrm{~b}}\right.$ $=3.6 \mathrm{~Hz}, \mathrm{H}-4 \mathrm{~b}), 4.80\left(2 \mathrm{H}, \mathrm{d}, J_{6 \mathrm{~b}, 5 \mathrm{~b}}=4.9 \mathrm{~Hz}, \mathrm{H}-6 \mathrm{~b}\right), 4.28(1 \mathrm{H}, \mathrm{bs}, \mathrm{H}-2 \mathrm{a}), 4.14\left(1 \mathrm{H}, \mathrm{dd}, J_{3 \mathrm{a}, 2 \mathrm{a}}=\right.$ $\left.3.3 \mathrm{~Hz}, J_{3 \mathrm{a}, 4 \mathrm{a}}=9.3 \mathrm{~Hz}, \mathrm{H}-3 \mathrm{a}\right), 4.02\left(1 \mathrm{H}\right.$, app. td, $\left.J_{4 \mathrm{a}, 5 \mathrm{a}}=9.6 \mathrm{~Hz}, J_{4 \mathrm{a}, \mathrm{OH}}=2.7 \mathrm{~Hz}, \mathrm{H}-4\right), 3.87$ $\left(1 \mathrm{H}, \mathrm{dd}, J_{6 \mathrm{a}, 6^{\mathrm{a}} \mathrm{a}}=11 \mathrm{~Hz}, J_{6 \mathrm{a}, 5 \mathrm{a}}=4.2 \mathrm{~Hz}, \mathrm{H}-6 \mathrm{a}\right), 3.81\left(1 \mathrm{H}, \mathrm{dd}, J_{6^{\mathrm{a}}, 5 \mathrm{a}}=5.4 \mathrm{~Hz}, \mathrm{H}-6^{\mathrm{a}} \mathrm{a}\right), 3.65-3.58$ $(1 \mathrm{H}, \mathrm{m}, \mathrm{H}-5 \mathrm{a}), 3.17(1 \mathrm{H}, \mathrm{d}, \mathrm{OH}), 2.94\left(1 \mathrm{H}, \mathrm{d}, J_{\mathrm{OH}, 2 \mathrm{a}}=2.5 \mathrm{~Hz}, \mathrm{OH}\right), 0.84\left(9 \mathrm{H}, \mathrm{s},{ }^{t} B u-\mathrm{Si}\right), 0.04$ $\left[6 \mathrm{H}, \mathrm{s}, \mathrm{OSi}\left(\mathrm{CH}_{3}\right)_{2}\right] .{ }^{13} \mathrm{C} \mathrm{NMR}\left(\mathrm{CDCl}_{3}\right): \delta_{\mathrm{C}} 166.3,166.2,165.7,165.6(\mathrm{COPh}), 160.8,142.6$ $\left(\mathrm{C}_{p \mathrm{NP}}\right), 133.8,133.7,133.4,133.2,130.2,129.9,129.8,129.4,129.3,128.8,128.5,128.4$ $\left(\mathrm{C}_{\mathrm{Ph}}\right), 125.8,116.5\left(\mathrm{C}_{p \mathrm{NP}}\right), 103.8(\mathrm{C}-1 \mathrm{~b}), 97.7(\mathrm{C}-1 \mathrm{a}), 83.1(\mathrm{C}-2 \mathrm{~b}), 81.2(\mathrm{C}-4 \mathrm{~b}), 77.3(\mathrm{C}-3 \mathrm{a})$, 76.8 (C-3b), 73.0 (C-5a), 70.1 (C-5b), 67.5 (C-2a), 66.8 (C-4a), 63.6 (C-6a), 63.3(C-6b), 25.8 $\left[\mathrm{SiC}\left(\mathrm{CH}_{3}\right)_{3}\right], 18.2\left[\mathrm{SiC}\left(\mathrm{CH}_{3}\right)_{3}\right],-5.5,-5.4\left[\mathrm{OSi}\left(\mathrm{CH}_{3}\right)_{2}\right]$. HRMS (ESI/Q-TOF) m/z: $[\mathrm{M}+\mathrm{Na}]^{+}$ Calcd for $\mathrm{C}_{52} \mathrm{H}_{55} \mathrm{NO}_{17} \mathrm{SiNa} 1016.3137$; Found 1016.3134.

6b: $[\alpha]_{\mathrm{D}}^{20}+65\left(\mathrm{c} 1, \mathrm{CH}_{2} \mathrm{Cl}_{2}\right) .{ }^{1} \mathrm{H} \mathrm{NMR}\left(\mathrm{CDCl}_{3}\right): \delta_{\mathrm{H}} 8.16\left(2 \mathrm{H}, \mathrm{d}, J=9.3 \mathrm{~Hz}, \mathrm{H}_{\mathrm{Ar}}\right), 7.97,7.93$, 7.86, $7.76\left(8 \mathrm{H}, 4 \mathrm{dd}, J=8.5 \mathrm{~Hz}, J=1.3 \mathrm{~Hz}, \mathrm{H}_{\mathrm{Ar}}\right), 7.61-7.30\left(12 \mathrm{H}, \mathrm{m}, \mathrm{H}_{\mathrm{Ar}}\right), 7.06(2 \mathrm{H}, \mathrm{d}, J=$ $\left.9.3 \mathrm{~Hz}, \mathrm{H}_{\mathrm{Ar}}\right), 6.47\left(1 \mathrm{H}, \mathrm{d}, J_{1 \mathrm{~b}, 2 \mathrm{~b}}=4.4 \mathrm{~Hz}, \mathrm{H}-1 \mathrm{~b}\right), 5.56\left(1 \mathrm{H}, \mathrm{dd}, J_{3 \mathrm{~b}, 4 \mathrm{~b}}=3.5 \mathrm{~Hz}, J_{3 \mathrm{~b}, 2 \mathrm{~b}}=0.9 \mathrm{~Hz}\right.$, H-3b), 5.47 (1H, d, $\left.J_{2 \mathrm{a}, 1 \mathrm{a}}=1.8 \mathrm{~Hz}, \mathrm{H}-1 \mathrm{a}\right), 5.37-5.32$ (1H, m, H-5b), 5.30 (1H, dd, H-2b), 4.69 $\left(1 \mathrm{H}, \mathrm{dd}, J_{4 \mathrm{~b}, 5 \mathrm{~b}}=8.0 \mathrm{~Hz}, \mathrm{H}-4 \mathrm{~b}\right), 4.56\left(1 \mathrm{H}, \mathrm{dd}, J_{6 \mathrm{~b}, 6^{\circ} \mathrm{b}}=12.5 \mathrm{~Hz}, J_{6 \mathrm{~b}, 5 \mathrm{~b}}=3.9 \mathrm{~Hz}, \mathrm{H}-6 \mathrm{~b}\right), 4.38(1 \mathrm{H}$, $\left.\mathrm{dd}, J_{6^{\circ} \mathrm{b}, 5 \mathrm{~b}}=4.9 \mathrm{~Hz}, \mathrm{H}-6^{`} \mathrm{~b}\right), 4.08\left(1 \mathrm{H}, \mathrm{dd}, J_{3 \mathrm{a}, 2 \mathrm{a}}=3,3 \mathrm{~Hz}, J_{3 \mathrm{a}, 4 \mathrm{a}}=9.4 \mathrm{~Hz}, \mathrm{H}-3 \mathrm{a}\right), 3.93(1 \mathrm{H}$, app.td, $\left.J_{4 \mathrm{a}, 5 \mathrm{a}}=9.5 \mathrm{~Hz}, J_{4 \mathrm{a}, \mathrm{OH}}=2 \mathrm{~Hz}, \mathrm{H}-4 \mathrm{a}\right), 3.78\left(2 \mathrm{H}, \mathrm{d}, J_{6 \mathrm{a}, 5 \mathrm{a}}=4.3 \mathrm{~Hz}, \mathrm{H}-6 \mathrm{a}\right), 3.64(1 \mathrm{H}, \mathrm{br} \mathrm{s}$, 
H-2a), 3.58-3.53 (1H, m, H-5a), $3.04(1 \mathrm{H}, \mathrm{d}, \mathrm{OH}), 2.37\left(1 \mathrm{H}, \mathrm{d}, J_{\mathrm{OH}, 2 \mathrm{a}}=2.8 \mathrm{~Hz}, \mathrm{OH}\right), 0.83(9 \mathrm{H}$, s, ${ }^{t} \mathrm{Bu}$-Si), $0.02\left[6 \mathrm{H}, \mathrm{s}, \mathrm{OSi}\left(\mathrm{CH}_{3}\right)_{2}\right] .{ }^{13} \mathrm{C} \mathrm{NMR}\left(\mathrm{CDCl}_{3}\right): \delta_{\mathrm{C}} 165.6,165.3,165.2(\mathrm{COPh}), 160.8$, $142.6\left(\mathrm{C}_{p \mathrm{NP}}\right), 136.1,133.7,133.4,133.2,133.1,130.0,129.9,129.8,129.6,129.5,129.4$, 128.6, 128.5, 128.3, 128.2, $125.8\left(\mathrm{C}_{\mathrm{Ph}}\right), 125.7\left(\mathrm{C}_{p \mathrm{NP}}\right), 123.3\left(\mathrm{PhCO}_{3}\right), 116.3\left(\mathrm{C}_{p \mathrm{NP}}\right), 105.3(\mathrm{C}-$ 1b), 97.4 (C-1a), 85.7 (C-2b), 83.9 (C-4b), 76.8 (C-3b), 73.7 (C-3a), 72.7 (C-5a), 71.2 (C-5b), 69.0 (C-2a), 67.3 (C-4a), 64.0 (C-6a), $62.8(\mathrm{C}-6 \mathrm{~b}), 25.8$ [SiC $\left.\left(\mathrm{CH}_{3}\right)_{3}\right], 18.3\left[\mathrm{SiC}\left(\mathrm{CH}_{3}\right)_{3}\right],-5.5$, 5.6 $\left[\mathrm{OSi}\left(\mathrm{CH}_{3}\right)_{2}\right]$. HRMS (ESI/Q-TOF) m/z: $[\mathrm{M}+\mathrm{Na}]^{+} \mathrm{Calcd}$ for $\mathrm{C}_{52} \mathrm{H}_{55} \mathrm{NO}_{17} \mathrm{SiNa} 1016.3137$; Found 1016.3130.

\section{4-Nitrophenyl $\quad 2,3,5,6$-tetra- $O$-benzoyl- $\beta$-D-galactofuranosyl-(1 $\rightarrow 3)-6$ - $O$-acetyl- $\alpha$-D-}

mannopyranoside (5c): Synthesized according to general procedure starting from 6-O-Acmannopyranoside $2 \mathbf{c}(52 \mathrm{mg}, 0.15 \mathrm{mmol})$, galactofuranosyl bromide 1a (200 mg, $0.30 \mathrm{mmol}$ ), 2-DPBA (34 mg, $0.15 \mathrm{mmol})$ and $\mathrm{Ag}_{2} \mathrm{O}(35 \mathrm{mg}, 0.15 \mathrm{mmol})$. Reaction reached completion after $2 \mathrm{~h}$. Purification by column chromatography on silica gel afforded $\mathbf{5 c}(119 \mathrm{mg}, 85 \%)$ as a white solid. $[\alpha]_{\mathrm{D}}{ }^{20}+37.5\left(\mathrm{c} 0.8, \mathrm{CH}_{2} \mathrm{Cl}_{2}\right) .{ }^{1} \mathrm{H}$ NMR $\left(\mathrm{CDCl}_{3}\right): \delta_{\mathrm{H}} 8.20\left(2 \mathrm{H}, \mathrm{d}, J=9.3 \mathrm{~Hz}, \mathrm{H}_{\mathrm{Ar}}\right)$, 8.11, 8.02, 7.99, $7.93\left(8 \mathrm{H}, 4 \mathrm{dd}, J=1.3 \mathrm{~Hz}, J=8.4 \mathrm{~Hz}, \mathrm{H}_{\mathrm{Ar}}\right), 7.60-7.32\left(12 \mathrm{H}, \mathrm{m}, \mathrm{H}_{\mathrm{Ar}}\right), 7.14$ $\left(2 \mathrm{H}, \mathrm{d}, \mathrm{H}_{\mathrm{Ar}}\right), 5.99-5.94(1 \mathrm{H}, \mathrm{m}, \mathrm{H}-5 \mathrm{~b}), 5.77\left(1 \mathrm{H}, \mathrm{dd}, J_{3 \mathrm{~b}, 2 \mathrm{~b}}=2.2 \mathrm{~Hz}, J_{3 \mathrm{~b}, 4 \mathrm{~b}}=6.0 \mathrm{~Hz}, \mathrm{H}-3 \mathrm{~b}\right)$, $5.71\left(1 \mathrm{H}, \mathrm{d}, J_{1 \mathrm{a}, 2 \mathrm{a}}=1.5 \mathrm{~Hz}, \mathrm{H}-1 \mathrm{a}\right), 5.54(1 \mathrm{H}, \mathrm{br} \mathrm{s}, \mathrm{H}-1 \mathrm{~b}), 5.49\left(1 \mathrm{H}, \mathrm{dd}, J_{2 \mathrm{~b}, 1 \mathrm{~b}}=0.7 \mathrm{~Hz}, \mathrm{H}-2 \mathrm{~b}\right)$, $4.95\left(1 \mathrm{H}, \mathrm{dd}, J_{4 \mathrm{~b}, 5 \mathrm{~b}}=3.7 \mathrm{~Hz}, \mathrm{H}-4 \mathrm{~b}\right), 4.83\left(1 \mathrm{H}, \mathrm{dd}, J_{6 \mathrm{~b}, 6^{\circ} \mathrm{b}}=11.9 \mathrm{~Hz}, J_{6 \mathrm{~b}, 5 \mathrm{~b}}=4.8 \mathrm{~Hz}, \mathrm{H}-6 \mathrm{~b}\right), 4.78$ $\left(1 \mathrm{H}, \mathrm{dd}, J_{6^{\mathrm{c}} \mathrm{b}, 5 \mathrm{~b}}=6.1 \mathrm{~Hz}, \mathrm{H}-6^{\mathrm{c}} \mathrm{b}\right), 4.39\left(1 \mathrm{H}, \mathrm{dd}, J_{6 \mathrm{a}, 5 \mathrm{a}}=5.2 \mathrm{~Hz}, J_{6 \mathrm{a}, 6^{\circ} \mathrm{a}}=12.2 \mathrm{~Hz}, \mathrm{H}-6 \mathrm{a}\right), 4.31$ $(1 \mathrm{H}$, br s, H-2a $), 4.27\left(1 \mathrm{H}, \mathrm{dd}, J_{66^{\mathrm{a}}, 5 \mathrm{a}}=2.2 \mathrm{~Hz}, \mathrm{H}-6{ }^{\circ} \mathrm{a}\right), 4.13\left(1 \mathrm{H}, \mathrm{dd}, J_{3 \mathrm{a}, 2 \mathrm{a}}=3.3 \mathrm{~Hz}, J_{3 \mathrm{a}, 4 \mathrm{a}}=9.3\right.$ Hz, H-3), $3.96\left(1 \mathrm{H}\right.$, app. td, $\left.J_{4 \mathrm{a}, \mathrm{OH}}=3.4 \mathrm{~Hz}, J_{4 \mathrm{a}, 5 \mathrm{a}}=9.7 \mathrm{~Hz}, \mathrm{H}-4 \mathrm{a}\right), 3.74(1 \mathrm{H}, \mathrm{ddd}, \mathrm{H}-5 \mathrm{a}), 3.24$ $(1 \mathrm{H}, \mathrm{d}, \mathrm{OH}), 3.08\left(1 \mathrm{H}, \mathrm{d}, J_{2 \mathrm{a}, \mathrm{OH}}=1.9 \mathrm{~Hz}, \mathrm{OH}\right), 2.04\left(3 \mathrm{H}, \mathrm{s}, \mathrm{CH} \mathrm{H}_{3} \mathrm{CO}\right) .{ }^{13} \mathrm{C} \mathrm{NMR}\left(\mathrm{CDCl}_{3}\right): \delta_{\mathrm{C}}$ $171.3\left(\mathrm{COCH}_{3}\right), 166.3,165.6(\mathrm{COPh}), 160.6,142.8,133.8,133.7,133.5,133.3,130.0,129.9$, $129.8,129.4,129.3,128.7,128.6,128.5,128.4,125.7,116.4\left(\mathrm{C}_{\mathrm{Ar}}\right), 103.9(\mathrm{C}-1 \mathrm{~b}), 97.8(\mathrm{C}-1 \mathrm{a})$, 83.3 (C-2b), 81.0 (C-4b), 77.0 (C-3a),76.7 (C-3b), 71.7 (C-5a), 70.0 (C-5b), 67.6 (C-2a), 65.3 
(C-4a), 63.1, 63.0 (C-6a, C-6b), $20.8\left(\mathrm{CH}_{3} \mathrm{CO}\right)$. HRMS (ESI/Q-TOF) m/z: $[\mathrm{M}+\mathrm{Na}]^{+} \mathrm{Calcd}$ for $\mathrm{C}_{48} \mathrm{H}_{43} \mathrm{NO}_{18} \mathrm{Na} 944.23723$; Found 944.2391.

\section{Octyl 2,3,5,6-O-benzoyl- $\beta$-D-galactofuranosyl)-(1 $\rightarrow 3)-6$ - $O$-acetyl- $\alpha$-D-mannopyranoside}

(8a): Synthesized according to general procedure starting from 6-O-Ac-mannopyranoside 4a (51 mg, $0.15 \mathrm{mmol})$, galactofuranosyl bromide 1a (200 mg, $0.30 \mathrm{mmol}), 2-D P B A(34 \mathrm{mg}$, $0.15 \mathrm{mmol})$ and $\operatorname{Ag}_{2} \mathrm{O}(35 \mathrm{mg}, 0.15 \mathrm{mmol})$. Reaction reached completion after $2 \mathrm{~h}$. Purification by column chromatography on silica gel afforded $8 \mathbf{a}(56 \mathrm{mg}, 40 \%)$ as a white solid. Further elution allowed isolating the corresponding orthoester 9a (68 mg, 49\%).

Alternative protocol using $4 \mathbf{a}(44 \mathrm{mg}, 0.13 \mathrm{mmol}), \mathbf{1 a}(176 \mathrm{mg}, 0.27 \mathrm{mmol}), 2-D P B A(30 \mathrm{mg}$, $0.13 \mathrm{mmol})$ and $\operatorname{AgOTf}(34 \mathrm{mg}, 0.13 \mathrm{mmol})$ as promoter gave $8 \mathbf{a}(70 \mathrm{mg}, 57 \%)$ in mixture with the octyl $\beta$-D-Galf-(1 $\rightarrow 4)-\mathrm{D}-\mathrm{Man} p$ regioisomer (ratio 4:1).

8a: $[\alpha]_{\mathrm{D}}{ }^{20}+6\left(\mathrm{c} 1.5, \mathrm{CHCl}_{3}\right) .{ }^{1} \mathrm{H} \mathrm{NMR}\left(\mathrm{CDCl}_{3}\right): \delta_{\mathrm{H}} 8.13-7.88(8 \mathrm{H}, \mathrm{m}, \mathrm{H} \mathrm{Ar}), 7.62-7.47(4 \mathrm{H}, \mathrm{m}$, $\left.\mathrm{H}_{\mathrm{Ar}}\right), 7.46-7.28\left(8 \mathrm{H}, \mathrm{m}, \mathrm{H}_{\mathrm{Ar}}\right), 5.96\left(1 \mathrm{H}, \mathrm{dt}, J_{5 \mathrm{~b}, 6^{\prime} \mathrm{b}}=6.2 \mathrm{~Hz}, J_{5 \mathrm{~b}, 6 \mathrm{~b}}=4.7 \mathrm{~Hz}, J_{5 \mathrm{~b}, 4 \mathrm{~b}}=3.8 \mathrm{~Hz}, \mathrm{H}-\right.$ $5 \mathrm{~b}), 5.73\left(1 \mathrm{H}, \mathrm{dd}, J_{3 \mathrm{~b}, 4 \mathrm{~b}}=5.8 \mathrm{~Hz}, J_{3 \mathrm{~b}, 2 \mathrm{~b}}=1.9 \mathrm{~Hz}, \mathrm{H}-3 \mathrm{~b}\right), 5.51-5.46(2 \mathrm{H}, \mathrm{m}, \mathrm{H}-1 \mathrm{~b}, \mathrm{H}-2 \mathrm{~b}), 4.91$ $(1 \mathrm{H}, \mathrm{dd}, \mathrm{H}-4 \mathrm{~b}), 4.89\left(1 \mathrm{H}, \mathrm{d}, J_{1 \mathrm{a}, 2 \mathrm{a}}=1.6 \mathrm{~Hz}, \mathrm{H}-1 \mathrm{a}\right), 4.80\left(1 \mathrm{H}, \mathrm{dd}, J_{6 \mathrm{~b}, 6^{\prime} \mathrm{b}}=11.9 \mathrm{~Hz}, \mathrm{H}-6 \mathrm{~b}\right), 4.74$ $\left(1 \mathrm{H}, \mathrm{dd}, \mathrm{H}-6{ }^{\prime} \mathrm{b}\right), 4.40\left(1 \mathrm{H}, \mathrm{dd}, J_{6 \mathrm{a}, 6{ }^{\prime} \mathrm{a}}=11.9 \mathrm{~Hz}, J_{6 \mathrm{a}, 5 \mathrm{a}}=4.7 \mathrm{~Hz}, \mathrm{H}-6 \mathrm{a}\right), 4.36\left(1 \mathrm{H}, \mathrm{dd}, J_{6}{ }^{\prime} \mathrm{a}, 5 \mathrm{a}=\right.$ $2.8 \mathrm{~Hz}, \mathrm{H}-6$ 'a), 4.07 (1H, dd, $\left.J_{2 \mathrm{a}, 3 \mathrm{a}}=3.3 \mathrm{~Hz}, \mathrm{H}-2 \mathrm{a}\right), 3.95\left(1 \mathrm{H}, \mathrm{dd}, J_{3 \mathrm{a}, 4 \mathrm{a}}=9.2 \mathrm{~Hz}, \mathrm{H}-3 \mathrm{a}\right), 3.86$ $\left(1 \mathrm{H}, \mathrm{dd}, J_{4 \mathrm{a}, 5 \mathrm{a}}=9.7 \mathrm{~Hz}, \mathrm{H}-4 \mathrm{a}\right), 3.77(1 \mathrm{H}, \mathrm{ddd}, \mathrm{H}-5 \mathrm{a}), 3.66\left(1 \mathrm{H}, \mathrm{dt},{ }^{2} J=9.7 \mathrm{~Hz},{ }^{3} J=6.8 \mathrm{~Hz}\right.$, $\left.\mathrm{OCH}_{2}\right), 3.42\left(1 \mathrm{H}, \mathrm{dt},{ }^{3} \mathrm{~J}=6.6 \mathrm{~Hz}, \mathrm{OCH}_{2}\right), 2.11\left(3 \mathrm{H}, \mathrm{s}, \mathrm{COCH}_{3}\right) 1.56\left(2 \mathrm{H}, \mathrm{m}, \mathrm{OCH}_{2} \mathrm{CH}_{2}\right)$, 1.36-1.20 (10H, m, $\left.\left(\mathrm{CH}_{2}\right)_{5} \mathrm{CH}_{3}\right), 0.87\left(3 \mathrm{H}, \mathrm{t},{ }^{3} \mathrm{~J}=6.9, \mathrm{CH}_{2} \mathrm{CH}_{3}\right) .{ }^{13} \mathrm{C} \mathrm{NMR}\left(\mathrm{CDCl}_{3}\right): \delta_{\mathrm{C}} 171.5$, $166.4,166.2,165.8,165.7$ (CO), 134.8, 133.8, 133.5, 133.3, 130.1, 130.1, 130.0, 129.9, 129.6, 129.5, 128.9, 128.7, 128.6, 128.6, 128.5, $128.0\left(\mathrm{C}_{\mathrm{Ar}}\right), 104.4(\mathrm{C}-1 \mathrm{~b}), 99.5(\mathrm{C}-1 \mathrm{a}), 83.1$ (C-2b), 81.2 (C-4b), 78.8 (C-3a), 77.0 (C-3b), 70.4 (C-5a), 70.3 (C-5b), 68.6 (C-2a), 68.1 $\left(\mathrm{OCH}_{2}\right), 66.0$ (C-4a), 63.8 (C-6a), 63.2 (C-6b), 32.0, 29.5, 29.5, 29.3, 26.2, $22.8\left[\left(\mathrm{CH}_{2}\right)_{6} \mathrm{CH}_{3}\right]$, 
$21.1\left(\mathrm{COCH}_{3}\right), 14.2\left(\mathrm{CH}_{2} \mathrm{CH}_{3}\right)$. HRMS (ESI/Q-TOF) m/z: [M+Na] Calcd for $\mathrm{C}_{50} \mathrm{H}_{56} \mathrm{O}_{16} \mathrm{Na}$ 935.34606; Found 935.3467.

9a: ${ }^{1} \mathrm{H}$ NMR $\left(\mathrm{CDCl}_{3}\right): \delta_{\mathrm{H}}$ 7.97-7.91 $\left(2 \mathrm{H}, \mathrm{m}, \mathrm{H}_{\mathrm{Ar}}\right), 7.91-7.83\left(4 \mathrm{H}, \mathrm{m}, \mathrm{H}_{\mathrm{Ar}}\right), 7.75-7.70(2 \mathrm{H}, \mathrm{m}$, $\left.\mathrm{H}_{\mathrm{Ar}}\right), 7.60-7.54\left(1 \mathrm{H}, \mathrm{m}, \mathrm{H}_{\mathrm{Ar}}\right), 7.53-7.46\left(2 \mathrm{H}, \mathrm{m}, \mathrm{H}_{\mathrm{Ar}}\right), 7.42-7.36\left(5 \mathrm{H}, \mathrm{m}, \mathrm{H}_{\mathrm{Ar}}\right), 7.36-7.28(4 \mathrm{H}$, $\left.\mathrm{m}, \mathrm{H}_{\mathrm{Ar}}\right), 6.36\left(1 \mathrm{H}, \mathrm{d}, J_{1 \mathrm{~b}, 2 \mathrm{~b}}=4.4 \mathrm{~Hz}, \mathrm{H}-1 \mathrm{~b}\right), 5.51\left(1 \mathrm{H}, \mathrm{dd}, J_{3 \mathrm{~b}, 4 \mathrm{~b}}=4.2 \mathrm{~Hz}, J_{3 \mathrm{~b}, 2 \mathrm{~b}}=1.5 \mathrm{~Hz}, \mathrm{H}-\right.$ 3b), $5.28\left(1 \mathrm{H}, \mathrm{ddd}, J_{5 \mathrm{~b}, 4 \mathrm{~b}}=7.6 \mathrm{~Hz}, J_{5 \mathrm{~b}, 6^{\circ} \mathrm{b}}=5.0 \mathrm{~Hz}, J_{5 \mathrm{~b}, 6 \mathrm{~b}}=3.9 \mathrm{~Hz}, \mathrm{H}-5 \mathrm{~b}\right), 5.23(1 \mathrm{H}, \mathrm{dd}, \mathrm{H}-2 \mathrm{~b})$, $4.71\left(1 \mathrm{H}, \mathrm{d}, J_{1 \mathrm{a}, 2 \mathrm{a}}=1.7 \mathrm{~Hz}, \mathrm{H}-1 \mathrm{a}\right), 4.62(1 \mathrm{H}, \mathrm{dd}, \mathrm{H}-4 \mathrm{~b}), 4.51\left(1 \mathrm{H}, \mathrm{dd}, J_{6 \mathrm{~b}, 6^{\circ} \mathrm{b}}=12.4 \mathrm{~Hz}, \mathrm{H}-6 \mathrm{~b}\right)$, $4.42\left(1 \mathrm{H}, \mathrm{dd}, J_{6 \mathrm{a}, 6^{\prime} \mathrm{a}}=12.1 \mathrm{~Hz}, J_{6 \mathrm{a}, 5 \mathrm{a}}=4.6 \mathrm{~Hz}, \mathrm{H}-6 \mathrm{a}\right), 4.33\left(1 \mathrm{H}, \mathrm{dd}, \mathrm{H}-6{ }^{\prime} \mathrm{b}\right), 4.27\left(1 \mathrm{H}, \mathrm{dd}, J_{6}{ }^{\prime} \mathrm{a}, 5 \mathrm{a}\right.$ $=2.1 \mathrm{~Hz}, \mathrm{H}-6$ 'a), $3.90\left(1 \mathrm{H}, \mathrm{dd}, J_{3 \mathrm{a}, 4 \mathrm{a}}=8.8 \mathrm{~Hz}, J_{3 \mathrm{a}, 2 \mathrm{a}}=3.3 \mathrm{~Hz}, \mathrm{H}-3 \mathrm{a}\right), 3.79-3.67(2 \mathrm{H}, \mathrm{m}, \mathrm{H}-4 \mathrm{a}$, H5a), 3.63-3.55 (2H, m, H-2a, $\left.\mathrm{OCH}_{2}\right), 3.33\left(1 \mathrm{H}, \mathrm{dt},{ }^{2} J=9.7 \mathrm{~Hz},{ }^{3} J=6.6 \mathrm{~Hz}, \mathrm{OCH}_{2}\right), 2.87$ $\left(1 \mathrm{H}, \mathrm{d}, J_{\mathrm{OH}, 4 \mathrm{a}}=2.8 \mathrm{~Hz}, \mathrm{OH}\right), 2.17\left(1 \mathrm{H}, \mathrm{d}, J_{\mathrm{OH}, 2 \mathrm{a}}=3.2 \mathrm{~Hz}, \mathrm{OH}\right), 2.09\left(3 \mathrm{H}, \mathrm{s}, \mathrm{COCH}_{3}\right), 1.56-$ $1.46\left(2 \mathrm{H}, \mathrm{m}, \mathrm{OCH}_{2} \mathrm{CH}_{2}\right), 1.31-1.23\left(10 \mathrm{H}, \mathrm{m},\left(\mathrm{CH}_{2}\right)_{5} \mathrm{CH}_{3}\right), 0.88\left(3 \mathrm{H}, \mathrm{t},{ }^{3} J=6.9 \mathrm{~Hz}\right.$, $\left.\left(\mathrm{CH}_{2}\right)_{5} \mathrm{CH}_{3}\right) .{ }^{13} \mathrm{C}$ NMR $\left(\mathrm{CDCl}_{3}\right): \delta_{\mathrm{C}} 171.7\left(\mathrm{COCH}_{3}\right), 165.8,165.4,165.3(\mathrm{COPh}), 136.6$, $133.8,133.2,133.2,130.1,130.0,129.9,129.8,129.6,128.7,128.6,128.5,128.4,126.2\left(\mathrm{C}_{\mathrm{Ar}}\right)$, $123.9\left(\mathrm{PhCO}_{3}\right), 105.1$ (C-1b), 99.3 (C-1a), 86.0 (C-2b), 83.1 (C-4b), 76.7 (C-3b), 74.5 (C-3a), 71.2 (C-5b), 70.6 (C-5a), $70.2(\mathrm{C}-2 \mathrm{a}), 68.0\left(\mathrm{OCH}_{2}\right), 65.6(\mathrm{C}-4 \mathrm{a}), 63.7$ (C-6a), 62.9 (C-6b), 31.9, 29.4, 29.4, 29.4, 26.2, $22.8\left[\left(\mathrm{CH}_{2}\right)_{6} \mathrm{CH}_{3}\right], 21.0\left(\mathrm{COCH}_{3}\right), 14.2\left[\left(\mathrm{CH}_{2}\right)_{6} \mathrm{CH}_{3}\right] . \mathrm{HRMS}$ (ESI/Q-TOF) m/z: [M+Na] $]^{+}$Calcd for $\mathrm{C}_{50} \mathrm{H}_{56} \mathrm{O}_{16} \mathrm{Na} 935.34606$; Found 935.3465.

\section{Thiotolyl}

\section{2,3,5,6-O-benzoyl- $\beta$-D-galactofuranosyl)-(1 $\rightarrow 3)-6-O$-acetyl- $\alpha-D-$}

mannopyranoside (8b): Synthesized according to general procedure starting from thiotolyl 6- $O$-Ac-mannopyranoside $4 \mathbf{b}(50 \mathrm{mg}, 0.15 \mathrm{mmol})$, galactofuranosyl bromide $\mathbf{1 a}(200 \mathrm{mg}$, $0.30 \mathrm{mmol}), 2-\mathrm{DPBA}(34 \mathrm{mg}, 0.15 \mathrm{mmol})$ and $\mathrm{Ag}_{2} \mathrm{O}$ (35 mg, $\left.0.15 \mathrm{mmol}\right)$. Reaction reached completion after 2 h. Purification by column chromatography on silica gel afforded $\mathbf{8 b}$ (131 $\mathrm{mg}, 96 \%$ ) as a white solid. 
Alternative protocol using $\mathbf{4 b}(50 \mathrm{mg}, 0.15 \mathrm{mmol}), \mathbf{1 a}(200 \mathrm{mg}, 0.30 \mathrm{mmol}), 2-\mathrm{DPBA}(34 \mathrm{mg}$, $0.14 \mathrm{mmol}$ ) and $\operatorname{AgOTf}(39 \mathrm{mg}, 0.15 \mathrm{mmol})$ as promoter gave $\mathbf{8 b}(94 \mathrm{mg}, 69 \%)$.

8b: $[\alpha]_{\mathrm{D}}^{20}+60\left(\mathrm{c} 1.15, \mathrm{CH}_{2} \mathrm{Cl}_{2}\right) .{ }^{1} \mathrm{H}$ NMR $\left(\mathrm{CDCl}_{3}\right): \delta_{\mathrm{H}} 8.11\left(2 \mathrm{H}, \mathrm{dd},{ }^{3} J=8.4 \mathrm{~Hz},{ }^{4} J=1.3 \mathrm{~Hz}\right.$, $\left.\mathrm{H}_{\mathrm{Ar}}\right), 8.02\left(4 \mathrm{H}, \mathrm{dd},{ }^{3} J=8.4 \mathrm{~Hz},{ }^{4} J=1.3 \mathrm{~Hz}, \mathrm{H}_{\mathrm{Ar}}\right), 7.93\left(2 \mathrm{H}, \mathrm{dd},{ }^{3} J=8.4 \mathrm{~Hz},{ }^{4} J=1.3 \mathrm{~Hz}, \mathrm{H}_{\mathrm{Ar}}\right)$, 7.61-7.51 (4H, m, H $\left.\mathrm{H}_{\mathrm{Ar}}\right), 7.46-7.31\left(10 \mathrm{H}, \mathrm{m}, \mathrm{H}_{\mathrm{Ar}}\right), 7.12\left(2 \mathrm{H}, \mathrm{d},{ }^{3} J=8.0 \mathrm{~Hz}, \mathrm{H}_{\mathrm{Tol}}\right), 5.94(1 \mathrm{H}, \mathrm{td}$, $\left.J_{5 \mathrm{~b}, 6 \mathrm{~b}}=5.3 \mathrm{~Hz}, J_{5 \mathrm{~b}, 4 \mathrm{~b}}=3.6 \mathrm{~Hz}, \mathrm{H}-5 \mathrm{~b}\right), 5.73\left(1 \mathrm{H}, \mathrm{dd}, J_{3 \mathrm{~b}, 4 \mathrm{~b}}=5.9 \mathrm{~Hz}, J_{3 \mathrm{~b}, 2 \mathrm{~b}}=2.2 \mathrm{~Hz}, \mathrm{H}-3 \mathrm{~b}\right), 5.54$ $\left(1 \mathrm{H}, \mathrm{d}, J_{1 \mathrm{a}, 2 \mathrm{a}}=1.4 \mathrm{~Hz}, \mathrm{H}-1 \mathrm{a}\right), 5.48\left(1 \mathrm{H}, \mathrm{d}, J_{1 \mathrm{~b}, 2 \mathrm{~b}}=0.8 \mathrm{~Hz}, \mathrm{H}-1 \mathrm{~b}\right), 5.48(1 \mathrm{H}, \mathrm{dd}, \mathrm{H}-2 \mathrm{~b}), 4.92$ $(1 \mathrm{H}, \mathrm{dd}, \mathrm{H}-4 \mathrm{~b}), 4.80(2 \mathrm{H}, \mathrm{d}, \mathrm{H}-6 \mathrm{~b}), 4.41\left(1 \mathrm{H}, \mathrm{dd}, J_{6 \mathrm{a}, 6{ }^{\mathrm{a}}}=12.1 \mathrm{~Hz}, J_{6 \mathrm{a}, 5 \mathrm{a}}=5.9 \mathrm{~Hz}, \mathrm{H}-6 \mathrm{a}\right), 4.37-$ 4.30 (3H, m, H-2a, H-5a, H-6'a), 3.98-3.88 (2H, m, H-3a, H-4a), 3.19 (1H, d, J $J_{\mathrm{OH}, 4 \mathrm{a}}=3.8 \mathrm{~Hz}$, $\mathrm{OH}), 2.33\left(3 \mathrm{H}, \mathrm{s}, \mathrm{CH}_{3}\right), 2.07\left(3 \mathrm{H}, \mathrm{s}, \mathrm{COCH}_{3}\right) .{ }^{13} \mathrm{C} \mathrm{NMR}\left(\mathrm{CDCl}_{3}\right): \delta_{\mathrm{C}} 171.4,166.5,166.2$, $165.8,165.7$ (CO), 138.1, 133.9, 133.6, 133.4, 132.5, 130.1, 130.0, 129.6, 129.5, 128.9, 128.7, 128.6 ( $\left.\mathrm{C}_{\mathrm{Ar}}\right), 104.1(\mathrm{C}-1 \mathrm{~b}), 87.8(\mathrm{C}-1 \mathrm{a}), 83.2(\mathrm{C}-2 \mathrm{~b}), 81.4(\mathrm{C}-4 \mathrm{~b}), 78.3$ (C-3a), 77.0 (C3b), 71.4 (C-5a), 70.2 (C-5b), 69.6 (C-2a), 66.4 (C-4a), 63.8 (C-6a), 63.2 (C-6b), $21.3\left(\mathrm{CH}_{3}\right)$, $21.0\left(\mathrm{COCH}_{3}\right)$. HRMS (ESI/Q-TOF) m/z: $[\mathrm{M}+\mathrm{Na}]^{+}$Calcd for $\mathrm{C}_{49} \mathrm{H}_{46} \mathrm{O}_{15} \mathrm{SNa} 929.24496$; Found 929.2455.

$p$-Nitrophenyl $(2,3,5,6-O$-tetrabenzoyl- $\beta$-D-galactofuranosyl)-(1 $\rightarrow 3)-6$ - $O$-acetyl- $\beta$-Dgalactopyranoside (17): Synthesized according to general procedure starting from $p \mathrm{NP}$ 6-OAc-galactopyranoside 12 (52 mg, $0.15 \mathrm{mmol})$, galactofuranosyl bromide 1a (200 mg, 0.30 mmol), 2-DPBA (34 mg, $0.15 \mathrm{mmol})$ and $\mathrm{Ag}_{2} \mathrm{O}(35 \mathrm{mg}, 0.15 \mathrm{mmol})$. Reaction reached completion after 2 h. Purification by column chromatography on silica gel afforded 17 (121 mg, $86 \%)$ as a white solid. $[\alpha]_{\mathrm{D}}{ }^{20}-26.5\left(\mathrm{c} 1, \mathrm{CHCl}_{3}\right) .{ }^{1} \mathrm{H}$ NMR $\left(\mathrm{CDCl}_{3}\right): \delta_{\mathrm{H}} 8.20(2 \mathrm{H}, \mathrm{d}, J=9.4$ $\left.\mathrm{Hz}, \mathrm{H}_{p \mathrm{NP}}\right), 8.12-8.06\left(2 \mathrm{H}, \mathrm{m}, \mathrm{H}_{\mathrm{Bz}}\right), 8.04-7.97\left(4 \mathrm{H}, \mathrm{m}, \mathrm{H}_{\mathrm{Bz}}\right), 7.95-7.89\left(2 \mathrm{H}, \mathrm{m}, \mathrm{H}_{\mathrm{Bz}}\right), 7.62-7.51$ $\left(3 \mathrm{H}, \mathrm{m}, \mathrm{H}_{\mathrm{Bz}}\right), 7.47-7.29\left(9 \mathrm{H}, \mathrm{m}, \mathrm{H}_{\mathrm{Bz}}\right), 7.12\left(2 \mathrm{H}, \mathrm{d}, \mathrm{H}_{p \mathrm{NP}}\right), 5.97\left(1 \mathrm{H}, \mathrm{ddd}, J_{5 \mathrm{~b}, 6^{\prime} \mathrm{b}}=6.3 \mathrm{~Hz}, J_{5 \mathrm{~b}, 6^{\circ} \mathrm{b}}\right.$ $\left.=5.0 \mathrm{~Hz}, J_{5 \mathrm{~b}, 4 \mathrm{~b}}=3.8 \mathrm{~Hz}, \mathrm{H}-5 \mathrm{~b}\right), 5.75\left(1 \mathrm{H}, \mathrm{dd}, J_{3 \mathrm{~b}, 4 \mathrm{~b}}=5.8 \mathrm{~Hz}, J_{3 \mathrm{~b}, 2 \mathrm{~b}}=2.2 \mathrm{~Hz}, \mathrm{H}-3 \mathrm{~b}\right), 5.69(1 \mathrm{H}$, $\left.\mathrm{d}, J_{1 \mathrm{~b}, 2 \mathrm{~b}}=0.9 \mathrm{~Hz} \mathrm{H}-1 \mathrm{~b}\right), 5.56(1 \mathrm{H}, \mathrm{dd}, \mathrm{H}-2 \mathrm{~b}), 4.99\left(1 \mathrm{H}, \mathrm{d}, J_{\mathrm{a} a, 2 \mathrm{a}}=7.8 \mathrm{~Hz}, \mathrm{H}-1 \mathrm{a}\right), 4.83(1 \mathrm{H}, \mathrm{dd}$, 
$\left.J_{6 \mathrm{~b}, 6^{\prime} \mathrm{b}}=11.8, \mathrm{H}-6 \mathrm{~b}\right), 4.80(1 \mathrm{H}, \mathrm{dd}, \mathrm{H}-4 \mathrm{~b}), 4.71(1 \mathrm{H}, \mathrm{dd}, \mathrm{H}-6$ 'b $), 4.34\left(1 \mathrm{H}, \mathrm{dd}, J_{6 \mathrm{a}, 6^{\prime} \mathrm{a}}=11.7 \mathrm{~Hz}\right.$,

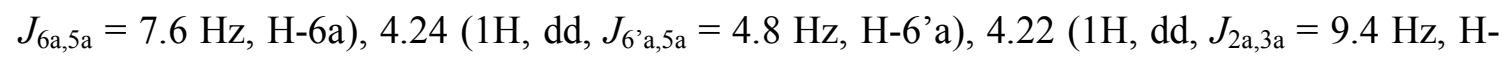
2a), $4.10\left(1 \mathrm{H}, \mathrm{dd}, J_{4 \mathrm{a}, 3 \mathrm{a}}=3.4 \mathrm{~Hz}, J_{4 \mathrm{a}, 5 \mathrm{a}}=1.1 \mathrm{~Hz}, \mathrm{H}-4 \mathrm{a}\right), 3.86(1 \mathrm{H}, \mathrm{ddd}, \mathrm{H}-5 \mathrm{a}), 3.79(1 \mathrm{H}, \mathrm{dd}$, H-3a), $2.08\left(3 \mathrm{H}, \mathrm{s}, \mathrm{CH}_{3}\right) .{ }^{13} \mathrm{C} \mathrm{NMR}\left(\mathrm{CDCl}_{3}\right): \delta_{\mathrm{C}} 170.8,166.3,166.3,165.8,165.7(\mathrm{COPh})$, $161.8\left(\mathrm{C}_{p \mathrm{NP}}\right), 142.9\left(\mathrm{C}_{p \mathrm{NP}}\right), 133.9,133.6,133.5,130.1,130.0,129.9,129.4,128.7,128.6$, $128.5\left(\mathrm{C}_{\mathrm{Bz}}\right), 125.7\left(\mathrm{C}_{p \mathrm{NP}}\right), 116.7\left(\mathrm{C}_{p \mathrm{NP}}\right), 107.9(\mathrm{C}-1 \mathrm{~b}), 100.2(\mathrm{C}-1 \mathrm{a}), 83.1(\mathrm{C}-2 \mathrm{~b}), 81.6(\mathrm{C}-4 \mathrm{~b})$, 80.7 (C-3a), 77.0 (C-3b), 72.9 (C-5a), 70.2 (C-2a, C-5b), 68.5 (C-4a), 63.0 (C-6a, C-6b), 20.9 $\left(\mathrm{CH}_{3}\right)$. HRMS (ESI/Q-TOF) m/z: [M+Na] ${ }^{+}$Calcd for $\mathrm{C}_{48} \mathrm{H}_{43} \mathrm{NO}_{18} \mathrm{Na} 944.23723$; Found 944.2368 .

$p$-Nitrophenyl $(2,3,5$, 6-tetra- $O$-benzoyl- $\beta$-D-galactofuranosyl)-(1 $\rightarrow 3)$-2-acetamido-6- $O$ acetyl-2-deoxy- $\beta$-D-galactopyranoside (18): Synthesized according to general procedure starting from $p$ NP 6-O-acetyl- $\beta-\mathrm{D}-N$-acetylgalactosamine 14 (58 $\mathrm{mg}, \quad 0.15 \mathrm{mmol}$ ), galactofuranosyl bromide 1a (200 mg, $0.30 \mathrm{mmol})$, 2-DPBA (34 mg, $0.15 \mathrm{mmol})$ and $\mathrm{Ag}_{2} \mathrm{O}$ (35 $\mathrm{mg}, 0.15 \mathrm{mmol}$ ). Reaction reached completion after $2 \mathrm{~h}$. Purification by column chromatography on silica gel afforded 18 (14 mg, 10\%) as a light brown solid. Further elution allowed isolating the corresponding orthoester 18' (54 mg, 37\%).

Alternative protocol using 14 (58 $\mathrm{mg}, 0.15 \mathrm{mmol}), \mathbf{1 a}(200 \mathrm{mg}, 0.30 \mathrm{mmol}), 2-\mathrm{DPBA}(34 \mathrm{mg}$, $0.15 \mathrm{mmol}$ ) and $\operatorname{AgOTf}(39 \mathrm{mg}, 0.15 \mathrm{mmol})$ as promoter gave 18 (80 $\mathrm{mg}, 55 \%)$ in mixture with the $p$ NP $\beta$-D-Galf-(1 $\rightarrow 4)$-D-GalNHAc regioisomer (ratio 4:1).

18: ${ }^{1} \mathrm{H}$ NMR $\left(\mathrm{CD}_{3} \mathrm{OD}+\mathrm{CDCl}_{3}\right): \delta_{\mathrm{H}} 8.15\left(2 \mathrm{H}, \mathrm{d},{ }^{3} J=9.2 \mathrm{~Hz}, \mathrm{H}_{p \mathrm{NP}}\right), 8.10-8.03\left(2 \mathrm{H}, \mathrm{m}, \mathrm{H}_{\mathrm{Bz}}\right)$, 8.02-7.93 $\left(4 \mathrm{H}, \mathrm{m}, \mathrm{H}_{\mathrm{Bz}}\right), 7.87-7.81\left(2 \mathrm{H}, \mathrm{m}, \mathrm{H}_{\mathrm{Bz}}\right), 7.57-7.50\left(3 \mathrm{H}, \mathrm{m}, \mathrm{H}_{\mathrm{Bz}}\right), 7.44-7.27(9 \mathrm{H}, \mathrm{m}$, $\left.\mathrm{H}_{\mathrm{Bz}}\right), 7.06\left(2 \mathrm{H}, \mathrm{d}, \mathrm{H}_{p \mathrm{NP}}\right), 5.95\left(1 \mathrm{H}, \mathrm{ddd}, J_{5 \mathrm{~b}, 6^{\prime} \mathrm{b}}=6.7 \mathrm{~Hz}, J_{5 \mathrm{~b}, 6 \mathrm{~b}}=4.4 \mathrm{~Hz}, J_{5 \mathrm{~b}, 4 \mathrm{~b}}=4.0 \mathrm{~Hz}, \mathrm{H}-5 \mathrm{~b}\right)$, $5.68\left(1 \mathrm{H}, \mathrm{d}, J_{1 \mathrm{a}, 2 \mathrm{a}}=8.2 \mathrm{~Hz}, \mathrm{H}-1 \mathrm{a}\right), 5.66\left(1 \mathrm{H}, \mathrm{dd}, J_{3 \mathrm{~b}, 4 \mathrm{~b}}=5.4 \mathrm{~Hz}, J_{3 \mathrm{~b}, 2 \mathrm{~b}}=1.9 \mathrm{~Hz}, \mathrm{H}-3 \mathrm{~b}\right), 5.43$ (1H, d, H-2b), 5.37 (1H, s, H-1b), 4.84 (1H, dd, H-4b), $4.76\left(1 \mathrm{H}, \mathrm{dd}, J_{6 \mathrm{~b}, 6^{\circ} \mathrm{b}}=11.8 \mathrm{~Hz}, \mathrm{H}-6 \mathrm{~b}\right)$, 
$4.67\left(1 \mathrm{H}, \mathrm{dd}, \mathrm{H}-6\right.$ 'b), $4.49\left(1 \mathrm{H}, \mathrm{dd}, J_{3 \mathrm{a}, 2 \mathrm{a}}=10.8 \mathrm{~Hz}, J_{3 \mathrm{a}, 4 \mathrm{a}}=3.3 \mathrm{~Hz}, \mathrm{H}-3 \mathrm{a}\right), 4.30\left(1 \mathrm{H}, \mathrm{dd}, J_{6 \mathrm{a}, 6}{ }^{\prime} \mathrm{a}\right.$ $\left.=11.7 \mathrm{~Hz}, J_{6 \mathrm{a}, 5 \mathrm{a}}=7.9 \mathrm{~Hz}, \mathrm{H}-6 \mathrm{a}\right), 4.16\left(1 \mathrm{H}, \mathrm{dd}, J_{6}{ }_{\mathrm{a}, 5 \mathrm{a}}=4.5 \mathrm{~Hz}, \mathrm{H}-6\right.$ 'a $), 4.09(1 \mathrm{H}, \mathrm{d}, \mathrm{H}-4 \mathrm{a})$, $3.89(1 \mathrm{H}, \mathrm{dd}, \mathrm{H}-5 \mathrm{a}), 3.80(1 \mathrm{H}, \mathrm{dd}, \mathrm{H}-2 \mathrm{a}), 2.03\left(3 \mathrm{H}, \mathrm{s}, \mathrm{CH}_{3}\right), 1.93\left(3 \mathrm{H}, \mathrm{s}, \mathrm{CH}_{3}\right) .{ }^{13} \mathrm{C} \mathrm{NMR}$ $\left(\mathrm{CD}_{3} \mathrm{OD}+\mathrm{CDCl}_{3}\right): \delta_{\mathrm{C}} 172.4,171.0\left(\mathrm{COCH}_{3}\right), 166.4,166.2,165.9,165.9(\mathrm{COPh}), 161.9$ $\left(\mathrm{C}_{p \mathrm{NP}}\right), 142.8\left(\mathrm{C}_{p \mathrm{NP}}\right), 133.9,133.6,133.5,130.1,130.0,129.9,129.8,129.4,128.7,128.6$, $128.5\left(\mathrm{C}_{\mathrm{Bz}}\right), 125.7\left(\mathrm{C}_{p \mathrm{NP}}\right), 116.7\left(\mathrm{C}_{p \mathrm{NP}}\right), 107.9(\mathrm{C}-1 \mathrm{~b}), 97.0(\mathrm{C}-1 \mathrm{a}), 83.0(\mathrm{C}-2 \mathrm{~b}), 81.2(\mathrm{C}-4 \mathrm{~b})$, 77.4 (C-3b), 76.8 (C-3a), 72.8 (C-5a), 70.3 (C-5b), 68.2 (C-4a), 63.3 (C-6a, C-6b), 53.4 (C2a), 23.3, $20.8\left(\mathrm{COCH}_{3}\right)$. HRMS (ESI/Q-TOF) m/z: $[\mathrm{M}+\mathrm{Na}]^{+}$Calcd for $\mathrm{C}_{50} \mathrm{H}_{46} \mathrm{~N}_{2} \mathrm{O}_{18} \mathrm{Na}$ 985.26378; Found 985.2646.

18': ${ }^{1} \mathrm{H}$ NMR $\left(\mathrm{CDCl}_{3}\right): \delta_{\mathrm{H}} 8.14\left(2 \mathrm{H}, \mathrm{d},{ }^{3} J=9.3 \mathrm{~Hz}, \mathrm{H}_{p \mathrm{NP}}\right), 7.93-7.88\left(2 \mathrm{H}, \mathrm{m}, \mathrm{H}_{\mathrm{Bz}}\right), 7.86-7.80$ (4H, m, $\left.\mathrm{H}_{\mathrm{Bz}}\right), 7.73-7.68\left(2 \mathrm{H}, \mathrm{m}, \mathrm{H}_{\mathrm{Bz}}\right), 7.59-7.46\left(3 \mathrm{H}, \mathrm{m}, \mathrm{H}_{\mathrm{Bz}}\right), 7.43-7.27\left(9 \mathrm{H}, \mathrm{m}, \mathrm{H}_{\mathrm{Bz}}\right), 7.04$ $\left(2 \mathrm{H}, \mathrm{d}, \mathrm{H}_{p \mathrm{NP}}\right), 6.21\left(1 \mathrm{H}, \mathrm{d}, J_{1 \mathrm{~b}, 2 \mathrm{~b}}=4.3 \mathrm{~Hz}, \mathrm{H}-1 \mathrm{~b}\right), 5.84\left(1 \mathrm{H}, \mathrm{d}, J_{1 \mathrm{a}, 2 \mathrm{a}}=8.3 \mathrm{~Hz}, \mathrm{H}-1 \mathrm{a}\right), 5.76(1 \mathrm{H}$, $\mathrm{d}, J=7.0, \mathrm{NH}), 5.40\left(1 \mathrm{H}, \mathrm{dd}, J_{3 \mathrm{~b}, 4 \mathrm{~b}}=5.4 \mathrm{~Hz}, J_{3 \mathrm{~b}, 2 \mathrm{~b}}=1.8 \mathrm{~Hz}, \mathrm{H}-3 \mathrm{~b}\right), 5.19\left(1 \mathrm{H}, \mathrm{ddd}, J_{5 \mathrm{~b}, 4 \mathrm{~b}}=7.0\right.$ $\left.\mathrm{Hz}, J_{5 \mathrm{~b}, 6^{\prime} \mathrm{b}}=5.3 \mathrm{~Hz}, J_{5 \mathrm{~b}, 6 \mathrm{~b}}=3.7 \mathrm{~Hz}, \mathrm{H}-5 \mathrm{~b}\right), 5.10(1 \mathrm{H}, \mathrm{dd}, \mathrm{H}-2 \mathrm{~b}), 4.63\left(1 \mathrm{H}, \mathrm{dd}, J_{3 \mathrm{a}, 2 \mathrm{a}}=10.6 \mathrm{~Hz}\right.$, $\left.J_{3 \mathrm{a}, 4 \mathrm{a}}=3.0 \mathrm{~Hz}, \mathrm{H}-3 \mathrm{a}\right), 4.51(1 \mathrm{H}, \mathrm{dd}, \mathrm{H}-4 \mathrm{~b}), 4.42\left(1 \mathrm{H}, \mathrm{dd}, J_{6 \mathrm{~b}, 6^{\circ} \mathrm{b}}=12.4 \mathrm{~Hz}, \mathrm{H}-6 \mathrm{~b}\right), 4.32(2 \mathrm{H}, \mathrm{d}$, $\left.J_{6 \mathrm{a}, 5 \mathrm{a}}=6.2 \mathrm{~Hz}, \mathrm{H}-6 \mathrm{a}\right), 4.24\left(1 \mathrm{H}, \mathrm{dd}, \mathrm{H}-6\right.$ 'b), $4.10\left(1 \mathrm{H}, \mathrm{t}, J_{4 \mathrm{a}, 3 \mathrm{a}}=J_{4 \mathrm{a}, \mathrm{OH}}=3.0 \mathrm{~Hz}, \mathrm{H}-4 \mathrm{a}\right), 3.92$ $(1 \mathrm{H}, \mathrm{t}, \mathrm{H}-5 \mathrm{a}), 3.54\left(1 \mathrm{H}, \mathrm{ddd}, J_{2 \mathrm{a}, \mathrm{NH}}=7.0 \mathrm{~Hz}, \mathrm{H}-2 \mathrm{a}\right), 2.46(1 \mathrm{H}, \mathrm{d}, \mathrm{OH}), 2.07\left(3 \mathrm{H}, \mathrm{s}, \mathrm{OCOCH}_{3}\right)$, $1.98\left(3 \mathrm{H}, \mathrm{s}, \mathrm{NHCOCH}{ }_{3}\right) .{ }^{13} \mathrm{C} \mathrm{NMR}\left(\mathrm{CDCl}_{3}\right): \delta_{\mathrm{C}} 171.9$ (NHCO), 170.9 (OCO), 165.8, 165.4 $(\mathrm{COPh}), 161.9\left(\mathrm{C}_{p \mathrm{NP}}\right), 142.9\left(\mathrm{C}_{p \mathrm{NP}}\right), 137.4,133.9,133.3,133.2,130.2,129.9,129.8,129.5$, 128.7, 128.6, 128.5, 128.4, $126.2\left(\mathrm{C}_{\mathrm{Ar}}\right), 125.8\left(\mathrm{C}_{p \mathrm{NP}}\right), 124.8\left(\mathrm{PhCO}_{3}\right), 116.7\left(\mathrm{C}_{p \mathrm{NP}}\right), 104.5(\mathrm{C}-$ 1b), 96.6 (C-1a), 86.4 (C-2b), 81.2 (C-4b), 76.4 (C-3b), 72.7 (C-5a), 71.0 (C-5b), 70.0 (C-3a), 68.2 (C-4a), 63.2 (C-6a), 62.7 (C-6b), 53.9 (C-2a), $24.0\left(\mathrm{NHCOCH}_{3}\right), 21.0\left(\mathrm{OCOCH}_{3}\right)$. HRMS (ESI/Q-TOF) m/z: [M+Na] ${ }^{+}$Calcd for $\mathrm{C}_{50} \mathrm{H}_{46} \mathrm{~N}_{2} \mathrm{O}_{18} \mathrm{Na} 985.26378$; Found 985.2632.

$p$-Nitrophenyl $\quad(2, \quad 3, \quad 5, \quad 6$-tetra- $O$-benzoyl- $\beta$-D-galactofuranosyl)-( $1 \rightarrow 3)$ - $\alpha$-Lrhamnopyranoside (19): Synthesized according to general procedure starting from $p$ NP $\alpha$-L- 
rhamnopyranoside 15 (43 $\mathrm{mg}, 0.15 \mathrm{mmol})$, galactofuranosyl bromide 1a (200 mg, 0.30 mmol), 2-DPBA (34 mg, $0.15 \mathrm{mmol})$ and $\mathrm{Ag}_{2} \mathrm{O}(35 \mathrm{mg}, 0.15 \mathrm{mmol})$. Reaction reached completion after 2 h. Purification by column chromatography on silica gel afforded 19 (58 $\mathrm{mg}, 45 \%$ ) as a light brown foam. Further elution allowed isolating the corresponding orthoester 19' (43 mg, 33\%).

Alternative protocol using 15 (43 mg, $0.15 \mathrm{mmol}), 1 \mathrm{a}(200 \mathrm{mg}, 0.30 \mathrm{mmol}), 2-\mathrm{DPBA}$ (34 mg, $0.15 \mathrm{mmol}$ ) and $\operatorname{AgOTf}(39 \mathrm{mg}, 0.15 \mathrm{mmol})$ as promoter gave $19(77 \mathrm{mg}, 59 \%)$ in mixture with the $p$ NP $\beta$-D-Galf-(1 $\rightarrow 4)-\mathrm{L}-\mathrm{Rha} p$ regioisomer (ratio 9:1).

19: $[\alpha]_{\mathrm{D}}^{20}-90\left(\mathrm{c} 0.8, \mathrm{CHCl}_{3}\right) .{ }^{1} \mathrm{H} \mathrm{NMR}\left(\mathrm{CDCl}_{3}\right): \delta_{\mathrm{H}} 8.20\left(2 \mathrm{H}, \mathrm{d},{ }^{3} J=9.3 \mathrm{~Hz}, \mathrm{H}_{p \mathrm{NP}}\right), 8.10(2 \mathrm{H}$, $\left.\mathrm{dd},{ }^{3} J=8.4 \mathrm{~Hz},{ }^{4} J=1.3 \mathrm{~Hz}, \mathrm{H}_{\mathrm{Bz}}\right), 8.05-7.90\left(6 \mathrm{H}, \mathrm{m}, \mathrm{H}_{\mathrm{Bz}}\right), 7.62-7.48\left(4 \mathrm{H}, \mathrm{m}, \mathrm{H}_{\mathrm{Bz}}\right), 7.47-7.40$ $\left(2 \mathrm{H}, \mathrm{m}, \mathrm{H}_{\mathrm{Bz}}\right), 7.39-7.31\left(6 \mathrm{H}, \mathrm{m}, \mathrm{H}_{\mathrm{Bz}}\right), 7.11\left(2 \mathrm{H}, \mathrm{d}, \mathrm{H}_{p \mathrm{NP}}\right), 5.94\left(1 \mathrm{H}, \mathrm{ddd}, J_{5 \mathrm{~b}, 6^{\circ} \mathrm{b}}=6.2 \mathrm{~Hz}, J_{5 \mathrm{~b}, 6 \mathrm{~b}}\right.$ $\left.=5.6 \mathrm{~Hz}, J_{5 \mathrm{~b}, 4 \mathrm{~b}}=3.5 \mathrm{~Hz}, \mathrm{H}-5 \mathrm{~b}\right), 5.78\left(1 \mathrm{H}, \mathrm{dd}, J_{3 \mathrm{~b}, 4 \mathrm{~b}}=6.2 \mathrm{~Hz}, J_{3 \mathrm{~b}, 2 \mathrm{~b}}=2.7 \mathrm{~Hz}, \mathrm{H}-3 \mathrm{~b}\right), 5.65(1 \mathrm{H}$, $\left.\mathrm{d}, J_{1 \mathrm{~b}, 2 \mathrm{~b}}=1.1 \mathrm{~Hz}, \mathrm{H}-1 \mathrm{~b}\right), 5.54(1 \mathrm{H}, \mathrm{dd}, \mathrm{H}-2 \mathrm{~b}), 5.49\left(1 \mathrm{H}, \mathrm{d}, J_{1 \mathrm{a}, 2 \mathrm{a}}=1.9 \mathrm{~Hz}, \mathrm{H}-1 \mathrm{a}\right), 4.87(1 \mathrm{H}, \mathrm{dd}$, $\left.J_{6 \mathrm{~b}, 6^{\prime} \mathrm{b}}=11.8 \mathrm{~Hz}, \mathrm{H}-6 \mathrm{~b}\right), 4.84(1 \mathrm{H}, \mathrm{dd}, \mathrm{H}-4 \mathrm{~b}), 4.69\left(1 \mathrm{H}, \mathrm{dd}, \mathrm{H}-6\right.$ 'b), $4.26\left(1 \mathrm{H}, \mathrm{dd}, J_{2 \mathrm{a}, 3 \mathrm{a}}=3.4\right.$ Hz, H-2a), $4.05\left(1 \mathrm{H}, \mathrm{dd}, J_{3 \mathrm{a}, 4 \mathrm{a}}=9.3 \mathrm{~Hz}, \mathrm{H}-3 \mathrm{a}\right), 3.81\left(1 \mathrm{H}, \mathrm{t}, J_{4 \mathrm{a}, 3 \mathrm{a}}=J_{4 \mathrm{a}, 5 \mathrm{a}}=9.3 \mathrm{~Hz}, \mathrm{H}-4 \mathrm{a}\right), 3.71$ $\left(1 \mathrm{H}, \mathrm{qd}, J_{5 \mathrm{a}, \mathrm{CH} 3}=6.2 \mathrm{~Hz}, \mathrm{H}-5 \mathrm{a}\right), 3.12(1 \mathrm{H}, \mathrm{s}, \mathrm{OH}), 2.86(1 \mathrm{H}, \mathrm{s}, \mathrm{OH}), 1.31\left(3 \mathrm{H}, \mathrm{d}, \mathrm{CH}_{3}\right) .{ }^{13} \mathrm{C}$ $\operatorname{NMR}\left(\mathrm{CDCl}_{3}\right): \delta_{\mathrm{C}} 166.6,166.5,165.8,165.7(\mathrm{COPh}), 161.0\left(\mathrm{C}_{p \mathrm{NP}}\right), 142.7\left(\mathrm{C}_{p \mathrm{NP}}\right), 134.9$, $134.0,133.9,133.7,133.5,130.2,130.1,130.0,129.9,129.4,128.7,128.7,128.7,128.6$, 128.5, $128.1(\mathrm{CPh}), 125.9\left(\mathrm{C}_{p \mathrm{NP}}\right), 116.4\left(\mathrm{C}_{p \mathrm{NP}}\right), 108.6(\mathrm{C}-1 \mathrm{~b}), 97.8(\mathrm{C}-1 \mathrm{a}), 83.9(\mathrm{C}-2 \mathrm{~b}), 81.0$ (C-3a,C-4b), 76.7 (C-3b), 71.3 (C-4a), 70.3 (C-2a), 70.1 (C-5b), 69.4 (C-5a), 63.0 (C-6b), 17.8. $\left(\mathrm{CH}_{3}\right)$. HRMS (ESI/Q-TOF) m/z: $[\mathrm{M}+\mathrm{Na}]^{+}$Calcd for $\mathrm{C}_{46} \mathrm{H}_{41} \mathrm{NO}_{16} \mathrm{Na} 886.23175$; Found 886.2322 .

19': ${ }^{1} \mathrm{H}$ NMR $\left(\mathrm{CDCl}_{3}\right): \delta_{\mathrm{H}} 8.14\left(2 \mathrm{H}, \mathrm{d},{ }^{3} J=9.2 \mathrm{~Hz}, \mathrm{H}_{p \mathrm{NP}}\right), 7.96-7.91\left(2 \mathrm{H}, \mathrm{m}, \mathrm{H}_{\mathrm{Bz}}\right), 7.89-7.84$ $\left(4 \mathrm{H}, \mathrm{m}, \mathrm{H}_{\mathrm{Bz}}\right), 7.79-7.73\left(2 \mathrm{H}, \mathrm{m}, \mathrm{H}_{\mathrm{Bz}}\right), 7.61-7.55\left(1 \mathrm{H}, \mathrm{m}, \mathrm{H}_{\mathrm{Bz}}\right), 7.54-7.48\left(2 \mathrm{H}, \mathrm{m}, \mathrm{H}_{\mathrm{Bz}}\right)$, 7.44- 
$7.30\left(9 \mathrm{H}, \mathrm{m}, \mathrm{H}_{\mathrm{Bz}}\right), 7.05\left(2 \mathrm{H}, \mathrm{d}, \mathrm{H}_{p \mathrm{NP}}\right), 6.36\left(1 \mathrm{H}, \mathrm{d}, J_{1 \mathrm{~b}, 2 \mathrm{~b}}=4.2 \mathrm{~Hz}, \mathrm{H}-1 \mathrm{~b}\right), 5.53-5.49(2 \mathrm{H}, \mathrm{m}$, H-1a, H-3b), 5.28-5.23 (1H, m, H-5b), $5.25\left(1 \mathrm{H}, \mathrm{dd}, J_{2 \mathrm{~b}, 3 \mathrm{~b}}=1.5 \mathrm{~Hz}, \mathrm{H}-2 \mathrm{~b}\right), 4.63(1 \mathrm{H}, \mathrm{dd}$, $\left.J_{4 \mathrm{~b}, 3 \mathrm{~b}}=7.5 \mathrm{~Hz}, J_{4 \mathrm{~b}, 5 \mathrm{~b}}=4.6 \mathrm{~Hz}, \mathrm{H}-4 \mathrm{~b}\right), 4.51\left(1 \mathrm{H}, \mathrm{dd}, J_{6 \mathrm{~b}, 6^{\prime} \mathrm{b}}=12.5 \mathrm{~Hz}, J_{6 \mathrm{~b}, 5 \mathrm{~b}}=3.7 \mathrm{~Hz}, \mathrm{H}-6 \mathrm{~b}\right)$, $4.32\left(1 \mathrm{H}, \mathrm{dd}, J_{6^{\prime} \mathrm{b}, 5 \mathrm{~b}}=5.3 \mathrm{~Hz}, \mathrm{H}-6^{\prime} \mathrm{b}\right), 4.06\left(1 \mathrm{H}, \mathrm{t}, J_{\mathrm{OH}, 2 \mathrm{a}}=J_{2 \mathrm{a}, 3 \mathrm{a}}=3.1 \mathrm{~Hz}, \mathrm{H}-2 \mathrm{a}\right), 4.00(1 \mathrm{H}, \mathrm{dd}$, $\left.J_{3 \mathrm{a}, 4 \mathrm{a}}=8.8 \mathrm{~Hz}, \mathrm{H}-3 \mathrm{a}\right), 3.69-3.57(2 \mathrm{H}, \mathrm{m}, \mathrm{H}-4 \mathrm{a}, \mathrm{H}-5 \mathrm{a}), 2.59(1 \mathrm{H}, \mathrm{d}, \mathrm{OH}), 2.52\left(1 \mathrm{H}, \mathrm{d}, J_{\mathrm{OH}, 4 \mathrm{a}}=\right.$ $2.4 \mathrm{~Hz}, \mathrm{OH}), 1.26\left(3 \mathrm{H}, \mathrm{d}, J_{\mathrm{CH} 3,5 \mathrm{a}}=1.0 \mathrm{~Hz}, \mathrm{CH}_{3}\right) .{ }^{13} \mathrm{C} \mathrm{NMR}\left(\mathrm{CDCl}_{3}\right): \delta_{\mathrm{C}} 165.8,165.5,165.4$ $(\mathrm{COPh}), 161.0\left(\mathrm{C}_{p \mathrm{NP}}\right), 142.7\left(\mathrm{C}_{p \mathrm{NP}}\right), 136.6,134.0,133.3,130.1,129.9,129.8,128.7,128.6$, 128.5, 128.4, $126.2\left(\mathrm{C}_{\mathrm{Bz}}\right), 125.9\left(\mathrm{C}_{p \mathrm{NP}}\right), 124.3\left(\mathrm{PhCO}_{3}\right), 116.3\left(\mathrm{C}_{p \mathrm{NP}}\right), 104.8(\mathrm{C}-1 \mathrm{~b}), 97.7(\mathrm{C}-$ 1a), 86.2 (C-2b), 82.4 (C-4b), 76.6 (C-3b), 74.4 (C-3a), 71.1 (C-5b), 70.5 (C-4a), 70.0 (C-5a), $69.7(\mathrm{C}-2 \mathrm{a}), 62.9$ (C-6b), $17.8\left(\mathrm{CH}_{3}\right)$. HRMS (ESI/Q-TOF) m/z: $[\mathrm{M}+\mathrm{Na}]^{+}$Calcd for $\mathrm{C}_{46} \mathrm{H}_{41} \mathrm{NO}_{16} \mathrm{Na} 886.23175$; Found 886.2318.

\section{4-Nitrophenyl} $2,3,5,6$-tetra- $O$-benzoyl- $\beta$-D-galactofuranosyl- $(1 \rightarrow 6)-\beta$-D-

galactofuranoside (20): Synthesized according to general procedure starting from $p$ NP $\beta$-Dgalactofuranoside 16 (20 mg, $67 \mu \mathrm{mol})$, galactofuranosyl bromide 1a (88 mg, $0.13 \mathrm{mmol})$, 2DPBA $(15 \mathrm{mg}, 67 \mu \mathrm{mol})$ and $\mathrm{Ag}_{2} \mathrm{O}(15 \mathrm{mg}, 67 \mu \mathrm{mol})$. Reaction reached completion after $2 \mathrm{~h}$. Purification by column chromatography on silica gel afforded 20 (7 mg, 12\%) as a white solid. Further elution allowed isolating the corresponding orthoester 20' (35 mg, 56\%).

Alternative protocol using 16 (20 mg, $67 \mu \mathrm{mol}), \mathbf{1 a}(88 \mathrm{mg}, 0.13 \mathrm{mmol}), 2-D P B A(15 \mathrm{mg}, 65$ $\mu \mathrm{mol})$ and $\operatorname{AgOTf}(16 \mathrm{mg}, 65 \mu \mathrm{mol})$ as promoter yielded a 7:1 inseparable mixture of 20 and $p$ NP $\beta$-D-Galf-(1 $\rightarrow 5)$-D-Gal $f(51 \%$ overall yield, $30 \mathrm{mg}, 34 \mu \mathrm{mol})$.

20: ${ }^{1} \mathrm{H}$ NMR $\left(\mathrm{CD}_{3} \mathrm{OD}\right): \delta_{\mathrm{H}} 8.11-8.06\left(4 \mathrm{H}, \mathrm{m}, \mathrm{H}_{p \mathrm{NP}}, \mathrm{H}_{\mathrm{Bz}}\right), 7.93-7.81\left(6 \mathrm{H}, 3 \mathrm{dd},{ }^{3} J=8.3 \mathrm{~Hz},{ }^{4} J\right.$ $\left.=1.2 \mathrm{~Hz}, \mathrm{H}_{\mathrm{Bz}}\right), 7.66\left(1 \mathrm{H}, \mathrm{td},{ }^{3} J=7.3 \mathrm{~Hz},{ }^{4} J=1.2 \mathrm{~Hz}, \mathrm{H}_{\mathrm{Bz}}\right), 7.56-7.22\left(11 \mathrm{H}, \mathrm{m}, \mathrm{H}_{\mathrm{Bz}}\right), 7.03$ $\left(2 \mathrm{H}, \mathrm{d},{ }^{3} J=9.3 \mathrm{~Hz}, \mathrm{H}_{p \mathrm{NP}}\right), 5.77\left(1 \mathrm{H}, \mathrm{ddd}, J_{5 \mathrm{~b}, 6 \mathrm{~b}}=7.1 \mathrm{~Hz}, J_{5 \mathrm{~b}, 4 \mathrm{~b}}=J_{5 \mathrm{~b}, 6^{\prime} \mathrm{b}}=3.5 \mathrm{~Hz}, \mathrm{H}-5 \mathrm{~b}\right), 5.61$ $\left(1 \mathrm{H}, \mathrm{d}, J_{1 \mathrm{a}, 2 \mathrm{a}}=2.4 \mathrm{~Hz}, \mathrm{H}-1 \mathrm{a}\right), 5.51\left(1 \mathrm{H}, \mathrm{d}, J_{3 \mathrm{~b}, 4 \mathrm{~b}}=4.7 \mathrm{~Hz}, \mathrm{H}-3 \mathrm{~b}\right), 5.41(1 \mathrm{H}, \mathrm{s}, \mathrm{H}-1 \mathrm{~b}), 5.39(1 \mathrm{H}$, 
s, H-2b), 4.60 (1H, dd, $\left.J_{6 b, 6 ’ b}=12.1 \mathrm{~Hz}, \mathrm{H}-6 \mathrm{~b}\right), 4.42-4.36$ (2H, m, H-6’b, H-4b), 4.34 (1H, dd, $\left.J_{4 \mathrm{a}, 3 \mathrm{a}}=7.1 \mathrm{~Hz}, J_{4 \mathrm{a}, 5 \mathrm{a}}=1.9 \mathrm{~Hz}, \mathrm{H}-4 \mathrm{a}\right), 4.27\left(1 \mathrm{H}, \mathrm{dd}, J_{3 \mathrm{a}, 2 \mathrm{a}}=4.9 \mathrm{~Hz}, \mathrm{H}-3 \mathrm{a}\right), 4.15(1 \mathrm{H}, \mathrm{dd}, \mathrm{H}-2 \mathrm{a})$, $4.04\left(1 \mathrm{H}, \mathrm{ddd}, J_{5 \mathrm{a}, 6^{\prime} \mathrm{a}}=8.6 \mathrm{~Hz}, J_{5 \mathrm{a}, 6 \mathrm{a}}=6.2 \mathrm{~Hz}, \mathrm{H}-5 \mathrm{a}\right), 3.92\left(1 \mathrm{H}\right.$, app. t., $\left.J_{6 \mathrm{a}, 6^{\prime} \mathrm{a}}=8.6 \mathrm{~Hz}, \mathrm{H}-6 \mathrm{a}\right)$, $3.63\left(1 \mathrm{H}, \mathrm{ddd}, \mathrm{H}-6\right.$ 'a). ${ }^{13} \mathrm{C} \mathrm{NMR}\left(\mathrm{CDCl}_{3}\right) \delta_{\mathrm{C}} 167.6,167.2,167.1,166.8(C \mathrm{OPh}), 163.4\left(\mathrm{C}_{p \mathrm{NP}}\right)$, $143.6\left(\mathrm{C}_{p \mathrm{NP}}\right), 134.9,134.7,134.6,134.4,131.1,130.8,130.7,130.4,130.3,129.9,129.7$, $129.5\left(\mathrm{C}_{\mathrm{Bz}}\right), 126.7,117.4\left(\mathrm{C}_{p \mathrm{NP}}\right), 107.6(\mathrm{C}-1 \mathrm{a}), 106.3(\mathrm{C}-1 \mathrm{~b}), 84.5(\mathrm{C}-4 \mathrm{a}), 83.4(\mathrm{C}-2 \mathrm{a}), 83.3$ (C-4b), 83.2 (C-2b), 79.0 (C-3b), 77.1 (C-3a), 71.7 (C-5b), 68.8 (C-5a), 67.4 (C-6a), 64.9 (C$6 b)$.

20': ${ }^{1} \mathrm{H}$ NMR $\left(\mathrm{CD}_{3} \mathrm{OD}\right) \delta_{\mathrm{H}} 8.14\left(2 \mathrm{H}, \mathrm{d},{ }^{3} J=9.3 \mathrm{~Hz}, \mathrm{H}_{p \mathrm{NP}}\right), 7.96,7.83,7.62\left(8 \mathrm{H}, 3 \mathrm{~d},{ }^{3} J=7.9\right.$ $\left.\mathrm{Hz}, \mathrm{H}_{\mathrm{Ar}}\right), 7.54-7.27\left(12 \mathrm{H}, \mathrm{m}, \mathrm{H}_{\mathrm{Ar}}\right), 7.18\left(2 \mathrm{H}, \mathrm{d}, \mathrm{H}_{p \mathrm{NP}}\right), 6.06\left(1 \mathrm{H}, \mathrm{d}, J_{1 \mathrm{~b}, 2 \mathrm{~b}}=4.2 \mathrm{~Hz}, \mathrm{H}-1 \mathrm{~b}\right), 5.64$ $\left(1 \mathrm{H}, \mathrm{d}, J_{1 \mathrm{a}, 2 \mathrm{a}}=1.8 \mathrm{~Hz}, \mathrm{H}-1 \mathrm{a}\right), 5.51\left(1 \mathrm{H}, \mathrm{d}, J_{3 \mathrm{~b}, 4 \mathrm{~b}}=3.3 \mathrm{~Hz}, \mathrm{H}-3 \mathrm{~b}\right), 5.43-5.38(1 \mathrm{H}, \mathrm{m}, \mathrm{H}-5 \mathrm{~b})$, $5.10(1 \mathrm{H}, \mathrm{d}, \mathrm{H}-2 \mathrm{~b}), 4.66-4.59(2 \mathrm{H}, \mathrm{m}, \mathrm{H}-4 \mathrm{~b}, \mathrm{H}-6 \mathrm{~b}), 4.43\left(1 \mathrm{H}, \mathrm{dd}, J_{6} \mathrm{~b}_{, 6 \mathrm{~b}}=12.4 \mathrm{~Hz}, J_{6^{\prime} \mathrm{b}, 5 \mathrm{~b}}=5.5\right.$ Hz, H-6’b), $4.27\left(1 \mathrm{H}, \mathrm{dd}, J_{2 \mathrm{a}, 3 \mathrm{a}}=4.0 \mathrm{~Hz}, \mathrm{H}-2 \mathrm{a}\right), 4.18\left(1 \mathrm{H}, \mathrm{dd}, J_{3 \mathrm{a}, 4 \mathrm{a}}=6.4 \mathrm{~Hz}, \mathrm{H}-3 \mathrm{a}\right), 4.11(1 \mathrm{H}$, dd, $\left.J_{4 \mathrm{a}, 5 \mathrm{a}}=2.5 \mathrm{~Hz}, \mathrm{H}-4 \mathrm{a}\right), 3.86\left(1 \mathrm{H}\right.$, app. td., $\left.J_{5 \mathrm{a}, 6 \mathrm{a}}=6.8 \mathrm{~Hz}, \mathrm{H}-5 \mathrm{a}\right), 3.51\left(1 \mathrm{H}, \mathrm{dd}, J_{6 \mathrm{a}, 6}{ }^{\prime} \mathrm{a}=9.4\right.$ Hz, H-6a), 3.40 (1H, dd, H-6'a). ${ }^{13} \mathrm{C}$ NMR $\left(\mathrm{CDCl}_{3}\right) \delta_{\mathrm{C}} 167.1,166.7,166.7(\mathrm{COPh}), 163.2$, $143.5\left(\mathrm{C}_{p \mathrm{NP}}\right), 137.2,134.7,134.2,130.7,130.6,130.5,130.5,130.3,130.1,129.6,129.4$, 129.3, 129.1, $127.5\left(\mathrm{C}_{\mathrm{Ar}}\right), 126.5\left(\mathrm{C}_{p \mathrm{NP}}\right), 124.6\left(\mathrm{CO}_{3} \mathrm{Ph}\right), 117.5\left(\mathrm{C}_{p \mathrm{NP}}\right), 107.5(\mathrm{C}-1 \mathrm{a}), 106.5(\mathrm{C}-$ 1b), 86.8 (C-2b), 85.2 (C-4a), 84.6 (C-4b), 83.3 (C-2a), 77.9, 77.8(C-3b, C-3a), 72.7 (C-5b), 69.2 (C-5a), 64.9 (C-6a), 64.0 (C-6b).

Acknowledgment. We are grateful to CNRS and the "Ministère de l'enseignement supérieur et de la recherche" for financial supports, We thanks the "Centre Regional des Mesures Physiques de l'Ouest (Université de Rennes 1)" for the registration of the mass spectra. This work was supported by the French Agence Nationale de la Recherche (ANR-10-BLAN0718). 
Supporting Information Available: Details of the computational calculations and ${ }^{1} \mathrm{H}$ and

${ }^{13} \mathrm{C}$ NMR spectra of all new compounds. This material is available free of charge via the Internet at http://pubs.acs.org.

\section{References.}

(1) Acosta-Serrano, A.; Hutchinson, C.; Nakayasu, E. S.; Almeida, I. C.; Carrington, M. In Trypanosomes- After the Genome; Barry, D., McCulloch, R., Mottram, J., Acosta-Serrano, A., Eds.; Horizon Bioscience: 2007, p 319-337.

(2) Cabezas, Y.; Legentil, L.; Robert-Gangneux, F.; Daligault, F.; Belaz, S.; Nugier-Chauvin, C.; Tranchimand, S.; Tellier, C.; Gangneux, J.-P.; Ferrieres, V. Org. Biomol. Chem. 2015, 13, 8393-8404.

(3) Cescutti, P. In Microbial Glycobiology Structure, Relevance and Applications; Moran, A. P., Ed.; Elsevier: London, 2009, p 93-108.

(4) Tarbell, J. M.; Cancel, L. M. J. Intern. Med. 2016, 280, 97-113.

(5) Richards, M. R.; Lowary, T. L. ChemBioChem 2009, 10, 1920-1938.

(6) Peltier, P.; Euzen, R.; Daniellou, R.; Nugier-Chauvin, C.; Ferrières, V. Carbohvd. Res. 2008, 343, 1897-1923.

(7) Eppe, G.; El Bkassiny, S.; Vincent, S. P. In Carbohydrates in Drug Design ans Discovery; Jiménez-Barbero, J., Cañada, J., Martin-Santamaria, S., Eds.; The Royal society of Chemistry: London, 2015; Vol. 43, p 209-241.

(8) Argunov, D. A.; Krylov, V. B.; Nifantiev, N. E. Org. Biomol. Chem. 2015, 13, $3255-3267$.

(9) Completo, G. C.; Lowary, T. L. L.Org.Chem. 2008, 73, 4513-4525.

(10) Gandolfi-Donadio, L.; Gallo-Rodriguez, C.; de Lederkremer, R. M. J. Org. Chem. 2002, 67, 4430-4435.

(11) Marino, C.; Baldoni, L. ChemBioChem 2014, 15, 188-204.

(12) Ruda, K.; Lindberg, J.; Garegg, P. J.; Oscarson, S.; Konradsson, P. J. Am. Chem.Soc. 2000, 122, 11067-11072.

(13) Goto, K.; Sawa, M.; Tamai, H.; Imamura, A.; Ando, H.; Ishida, H.; Kiso, M. Chem. Eur. J. 2016, 22, 8323-8331.

(14) Poulin, M. B.; Lowary, T. L. J. Org Chem. 2016, 81, 8123-8130.

(15) Yamatsugu, K.; Splain, R. A.; Kiessling, L. L. J.Am. Chem. Soc. 2016, 138, 9205-9211.

(16) Chlubnova, I.; Kralova, B.; Dvorakova, H.; Hosek, P.; Spiwok, V.; Filipp, D.; Nugier-Chauvin, C.; Daniellou, R.; Ferrieres, V. Org. Biomol. Chem. 2014, 12, 3080-3089.

(17) Pennec, A.; Daniellou, R.; Loyer, P.; Nugier-Chauvin, C.; Ferrieres, V. Carbohvdr. Res. 2015, 402, 50-55.

(18) Kaji, E.; Shibayama, K.; In, K. Tetrahedron Lett. 2003, 44, 4881-4885.

(19) Maggi, A.; Madsen, R. Eur. J. Org. Chem. 2013, 2683-2691.

(20) Oshima, K.; Aoyama, Y. J. Am. Chem. Soc. 1999, 121, 2315-2316.

(21) Kaji, E.; Yamamoto, D.; Shirai, Y.; Ishige, K.; Arai, Y.; Shirahata, T.; Makino, K.; Nishino, T. Eur. J. Org. Chem. 2014, 3536-3539.

(22) Lee, D.; Taylor, M. S. J.Am. Chem. Soc. 2011, 133, 3724-3727.

(23) Chan, L.; Taylor, M. S. Org. Lett. 2011, 13, 3090-3093. 
(24) Lee, D.; Williamson, C. L.; Chan, L.; Taylor, M. S. J. Am. Chem. Soc. 2012, $134,8260-8267$.

(25) D'Angelo, K. A.; Taylor, M. S. J.Am. Chem. Soc. 2016, 138, 11058-11066.

(26) Euzen, R.; Guégan, J. P.; Ferrières, V.; Plusquellec, D. L.Org. Chem. 2007, 72, 5743-5747.

(27) Pedersen, L.; Turco, S. Cell. Mol.Life Sci. 2003, 60, 259-266.

(28) Lowary, T. L. Acc. Chem.Res. 2016, 49, 1379-1388.

(29) Gouliaras, C.; Lee, D.; Chan, L.; Taylor, M. S. J.Am.Chem.Soc. 2011, 133, 13926-13929.

(30) Marino, C.; Varela, O.; de Lederkremer, R. M. Tetrahedron 1997, 53, 1600916016.

(31) Ferrières, V.; Roussel, M.; Gelin, M.; Plusquellec, D. J. Carbohydr. Chem. 2001, 20, 855-865. 245-248.

(32) Gallo-Rodriguez, C.; Gandolfi, L.; de Lederkremer, R. M. Org. Lett. 1999, 1,

(33) Yang, W.; Mortier, W. J. J.Am. Chem. Soc. 1986, 108, 5708-5711.

(34) Fraser-Reid, B. L. In Handbook of Chemical Glycosylation: Advances in Stereoselectivity and Therapeutic Relevance; Demchenko, A. V., Ed.; Wiley-VCH: Weiheim, 2008, p 381-415.

(35) Gorin, P. A. J. Can. J. Chem. 1962, 40, 275-282.

(36) Abronina, P. I.; Sedinkin, S. L.; Podvalnyy, N. M.; Fedina, K. G.; Zinin, A. I.; Torgov, V. I.; Kononov, L. O. Tetrahedron Lett. 2011, 52, 1794-1796.

(37) Simerská, P.; Kuzma, M.; Pišvejcová, A.; Weignerová, L.; Macková, M.; Riva, S.; Křen, V. Eolia Microbiol.(Praha) 2003, 48, 329-337.

(38) Simerská, P.; Pišvejcová, A.; Kuzma, M.; Sedmera, P.; Křen, V. r.; Nicotra, S.; Riva, S. L.Mol.Catal. B: Enzvm. 2004, 29, 219-225.

(39) Chen, H.-M.; Withers, S. G. Carbohvdr. Res. 2007, 342, 2212-2222.

(40) Poláková, M.; Beláňová, M.; Petruš, L.; Mikušová, K. Carbohydr. Res. 2010, $345,1339-1347$.

(41) Watt, J. A.; Williams, S. J. Org Biomol. Chem. 2005, 3, 1982-1992. 